JOURNAL OF THE

AMERICAN MATHEMATICAL SOCIETY

Volume 9, Number 4, October 1996

\title{
VANISHING CYCLES FOR NON-ARCHIMEDEAN ANALYTIC SPACES
}

\author{
VLADIMIR G. BERKOVICH
}

\section{INTRODUCTION}

In this work we develop a formalism of vanishing cycles for non-Archimedean analytic spaces which is an analog of that for complex analytic spaces from [SGA7], Exp. XIV. As an application we prove that in the equicharacteristic case the stalks of the vanishing cycles sheaves of a scheme $\mathcal{X}$ at a closed point $\mathrm{x} \in \mathcal{X}_{s}$ depend only on the formal completion $\operatorname{Spf}\left(\widehat{\mathcal{O}}_{\mathcal{X}, \mathbf{x}}\right)$ of $\mathcal{X}$ at $\mathbf{x}$. In particular, any continuous homomorphism $\widehat{\mathcal{O}}_{\mathcal{X}, \mathbf{x}} \rightarrow \widehat{\mathcal{O}}_{\mathcal{Y}, \mathbf{y}}$ induces a homorphism from the stalks of the vanishing cycles sheaves of $\mathcal{X}$ at $\mathbf{x}$ to those of $\mathcal{Y}$ at $\mathbf{y}$. Furthermore, we prove that, given $\widehat{\mathcal{O}}_{\mathcal{X}, \mathbf{x}}$ and $\widehat{\mathcal{O}}_{\mathcal{Y}, \mathbf{y}}$, there exists $n \geq 1$ such that, for any pair of continuous homomorphisms $\widehat{\mathcal{O}}_{\mathcal{X}, \mathbf{x}} \rightarrow \widehat{\mathcal{O}}_{\mathcal{Y}, \mathbf{y}}$ that coincide modulo the $n$-th power of the maximal ideal of $\widehat{\mathcal{O}}_{\mathcal{Y}, \mathbf{y}}$, the induced homomorphisms between the stalks of the vanishing cycles sheaves coincide. These facts generalize a result of G. Laumon from [Lau] (see Remark 7.6).

Throughout the paper we fix a non-Archimedean field $k$ (whose valuation is not assumed to be nontrivial). In $\S 1$ we study étale Galois sheaves on $k$-analytic spaces. To define the vanishing cycles functor and to work with it, we use the language of pro-analytic spaces, i.e., pro-objects of the category of analytic spaces ([SGA4], Exp. I). Examples of such objects are the germs of analytic spaces as in [Ber2], $\S 3.4$. Another example is considered in $\S 3$. In $\S 4$ we define the vanishing cycles functor and establish its basic properties. In $\S 5$ we show that the vanishing cycles sheaves are trivial for smooth morphisms. In $\S 6$ we prove a comparison theorem for vanishing cycles. This theorem is more general than its analog over $\mathbf{C}$ from [SGA7], Exp. XIV, and its proof does not use Hironaka's theorem on resolution of singularities. In $\S 7$ we apply the comparison theorem to prove the properties of the vanishing cycles sheaves of schemes formulated above. It is worthwhile to note that this application is obtained by considering non-Archimedean analytic geometry over fields with trivial valuation.

Like [Ber3], this work arose from a suggestion of P. Deligne to apply the étale cohomology theory from [Ber2] to the study of the vanishing cycles sheaves of schemes. I am very grateful to him for useful discussions on the subject. I also

Received by the editors July 11, 1993.

1991 Mathematics Subject Classification. Primary 14F20, 32P05, 32S30.

Key words and phrases. Algebraic geometry, étale cohomology, p-adic analytic spaces.

The author is an Incumbent of the Reiter Family Career Development Chair.

Supported in part by NSF grant DMS-9100383. 
gratefully appreciate the hospitality and support of the Institute for Advanced Study, where a part of this work was done.

\section{$\S 1$. Galois sheaves}

For a $k$-analytic space $X$ and an algebraic extension $K$ over $k$ we set $X_{K}=X \widehat{\otimes} \widehat{K}$ and denote by $\pi=\pi_{X}$ the canonical morphism $X_{K} \rightarrow X$.

1.1. Lemma. Any $K$-analytic space $V$ étale over $X_{K}$ admits an étale covering $\left\{U_{K}^{i}\right\}_{i \in I}$ for some $U^{i}$ étale over $X$.

Proof. We have to verify that each point $v \in V$ has an étale neighborhood $U_{K}$ for some $U$ étale over $X$. Let $x^{\prime}$ be the image of $v$ in $X_{K}$ and set $x=\pi\left(x^{\prime}\right)$. Then the field $\mathcal{H}(v)$ is a finite separable extension of $\mathcal{H}\left(x^{\prime}\right)$. Since the compositum $\mathcal{H}(x) K$ is everywhere dense in $\mathcal{H}\left(x^{\prime}\right)$, it follows that $\mathcal{H}(v)=\mathcal{H}\left(x^{\prime}\right) L$ for some finite separable extension $L$ of $\mathcal{H}(x)$. By Theorem 3.4.1 from [Ber2], the field $L$ gives rise to an étale morphism of $k$-germs $(U, u) \rightarrow(X, x)$ with $\mathcal{H}(u)=L$. By construction, there exists a point $u^{\prime} \in U_{K}$ with $\mathcal{H}\left(u^{\prime}\right)=\mathcal{H}\left(x^{\prime}\right) L=\mathcal{H}(v)$, and therefore, by the same theorem, there is an isomorphism of $K$-germs $\left(U_{K}, u^{\prime}\right) \stackrel{\sim}{\longrightarrow}(V, v)$ over $\left(X_{K}, x^{\prime}\right)$. Thus, replacing $X$ by $U$, we may assume that $V$ is an open neighborhood of $x^{\prime}$ in $X_{K}$. It suffices to know that there exist a finite separable extension $k^{\prime}$ of $k$ in $K$ and an open neighborhood $U$ of the image of $x^{\prime}$ in $X_{k^{\prime}}$ such that the preimage of $U$ in $X_{K}$ is contained in $V$. But this is established in the proof of Lemma 5.3.4 from [Ber2].

Suppose that $K$ is a normal extension of $k$. Then the Galois group $G(K / k)$ acts on $X_{K}$ (considered as an analytic space over $k$ ). Let $\nu: G \rightarrow G(K / k)$ be a continuous homomorphism from a profinite group $G$ to $G(K / k)$. The group $G$ acts on $X_{K}$ via $\nu$. An action of $G$ on a sheaf of sets $F$ on $X_{K}$, compatible with the action of $G$ on $X_{K}$, is a system of isomorphisms $\sigma(g): \nu(g)_{*} F \rightarrow F, g \in G$, such that $\sigma(g h)=\sigma(g) \circ \nu(g)_{*}(\sigma(h))$. If $G$ acts on $F$, then for any $U$ étale over $X$ the group $G$ acts on the set $F\left(U_{K}\right)$.

1.2. Lemma. The following properties of an action of $G$ on $F$ are equivalent:

(a) for any $f \in F\left(U_{K}\right)$, where $U$ is étale over $X$, there exists an open covering $\left\{\mathcal{U}^{i}\right\}_{i \in I}$ of $U$ such that for any $i \in I$ the stabilizer of $\left.f\right|_{\mathcal{U}_{K}^{i}}$ is open in $G$;

(b) for any $f \in F\left(U_{K}\right)$, where $U$ is étale over $X$, there exists an étale covering $\left\{U^{i}\right\}_{i \in I}$ of $U$ such that for any $i \in I$ the stabilizer of $\left.f\right|_{U_{K}^{i}}$ is open in $G$.

Proof. Let $f \in F\left(U_{K}\right)$, where $U$ is étale over $X$, and suppose that there is an étale covering $\left\{U_{i} \stackrel{\varphi_{i}}{\rightarrow} U\right\}_{i \in I}$ such that for any $i \in I$ the stabilizer of $\left.f\right|_{U_{K}^{i}}$ is open in $G$. Since étale morphisms are quasifinite (see [Ber2], §3), we can replace the covering by a refinement so that all of the induced morphisms $U^{i} \rightarrow \mathcal{U}^{i}=\varphi_{i}\left(U^{i}\right)$ are finite. In this case the morphisms $U_{K}^{i} \rightarrow \mathcal{U}_{K}^{i}$ are finite and étale, and therefore the maps $F\left(\mathcal{U}_{K}^{i}\right) \rightarrow F\left(U_{K}^{i}\right)$ are injective. We get an open covering $\left\{\mathcal{U}^{i}\right\}_{i \in I}$ of $U$ such that for any $i \in I$ the stabilizer of $\left.f\right|_{\mathcal{U}_{K}^{i}}$ is open in $G$.

An action of $G$ on $F$, which possesses the equivalent properties of Lemma 1.2, is said to be continuous. Let $\mathbf{T}_{G}\left(X_{K}\right)$ (resp. $\mathbf{S}_{G}\left(X_{K}\right)$ ) denote the category of sheaves of sets (resp. abelian groups) endowed with a continuous action of $G$ (G-sheaves). The category $\mathbf{S}_{G}\left(X_{K}\right)$ has injectives. Its derived category will be 
denoted by $D_{G}\left(X_{K}\right)$. If $I$ is a subgroup of $G$ which is contained in the kernel of $\nu: G \rightarrow G(K / k)$, then $\nu$ induces a continuous homomorphism $\nu^{\prime}: G / I \rightarrow G(K / k)$, and one has a left exact functor $\mathbf{S}_{G}\left(X_{K}\right) \rightarrow \mathbf{S}_{G / I}\left(X_{K}\right): F \rightarrow F^{I}$. The values of its right derived functors are denoted by $\mathcal{H}^{q}(I, F)$. For $x^{\prime} \in X_{K}$ one has $\mathcal{H}^{q}(I, F)_{x^{\prime}}=$ $H^{q}\left(I, F_{x^{\prime}}\right)$.

Suppose that $k^{\prime}$ is another non-Archimedean field, $K^{\prime}$ is a normal extension of $k^{\prime}$, and $\nu^{\prime}: G^{\prime} \rightarrow G\left(K^{\prime} / k^{\prime}\right)$ is a continuous homomorphism of profinite groups. Furthermore, suppose we are given a commutative diagram of isometric embeddings

$$
\begin{array}{ccc}
K & \hookrightarrow & K^{\prime} \\
\uparrow & & \uparrow \\
k & \hookrightarrow & k^{\prime}
\end{array}
$$

and a commutative diagram of continuous homomorphisms

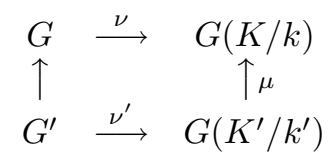

where $\mu$ is induced by the above embeddings. Finally, let $X^{\prime}$ be a $k^{\prime}$-analytic space, and let $\varphi: X^{\prime} \rightarrow X$ be a morphism over the embedding $k \hookrightarrow k^{\prime}$. It induces a morphism $\bar{\varphi}: X_{K^{\prime}}^{\prime} \rightarrow X_{K}$ over the embedding $\widehat{K} \hookrightarrow \widehat{K^{\prime}}$.

1.3. Lemma. The inverse image functor for the morphism $\bar{\varphi}$ induces a well defined functor $\bar{\varphi}^{*}: \mathbf{T}_{G}\left(X_{K}\right) \rightarrow \mathbf{T}_{G^{\prime}}\left(X_{K^{\prime}}^{\prime}\right)$.

Proof. Let $F \in \mathbf{T}_{G}\left(X_{K}\right)$ and $f \in\left(\bar{\varphi}^{*} F\right)\left(V_{K^{\prime}}\right)$, where $V$ is étale over $X^{\prime}$. Then there is an étale covering $\left\{W^{i} \rightarrow V_{K^{\prime}}\right\}_{i \in I}$ and, for each $i \in I$, a commutative diagram

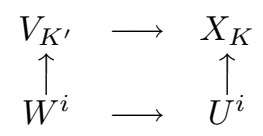

where $U^{i}$ is étale over $X_{K}$, such that $\left.f\right|_{W^{i}}$ is the image of some element $g_{i} \in F\left(U^{i}\right)$. By Lemma 1.1, we can replace the covering $\left\{W^{i}\right\}_{i \in I}$ by a refinement and assume that $W^{i}=V_{K^{\prime}}^{i}$ for some étale covering $\left\{V^{i}\right\}_{i \in I}$ of $V$. Furthermore, by the same lemma, we can find for each $i \in I$ an étale covering $\left\{U_{K}^{i j}\right\}_{j \in J_{i}}$ of $U^{i}$, where $U^{i j}$ are étale over $X$. Since the action of $G$ on $\mathcal{G}$ is continuous, we can replace the latter covering by a refinement and assume that the stabilizer of $\left.g_{i}\right|_{U_{K}^{i j}}$ is open in $G$. We get an étale covering $\left\{V^{i j}=V^{i} \times_{X} U^{i j}\right\}_{i, j}$ of $V$ such that $\left.f\right|_{V_{K^{\prime}}^{i j}}$ is the image of $\left.g_{i}\right|_{U_{K}^{i j}}$. It follows that the stabilizer of $\left.f\right|_{V_{K^{\prime}}^{i j}}$ is open in $G^{\prime}$.

From Lemma 1.3 it follows that the inverse image functor for the morphism $\pi: X_{K} \rightarrow X$ induces a well defined functor $\pi^{*}: \mathbf{T}(X) \rightarrow \mathbf{T}_{G}\left(X_{K}\right)$. In the case $G \stackrel{\sim}{\longrightarrow} G(K / k)$ one can easily show that there is an equivalence of categories $\pi^{*}: \mathbf{T}(X) \stackrel{\sim}{\longrightarrow} \mathbf{T}_{G(K / k)}\left(X_{K}\right)$.

\section{§2. Pro-analytic spaces}

Recall that the category of pro-objects of a category $C, \operatorname{Pro}(C)$, is defined as follows (see [SGA4], Exp. I). Its objects are functors $I \rightarrow C: i \mapsto X_{i}$, where $I$ is 
a small cofiltered category. Such an object is denoted by $\underset{I}{\lim } " X_{i}$. Morphisms are defined by

$$
\operatorname{Hom}(\underbrace{\lim }_{J} " Y_{j}, " \underset{I}{\lim } " X_{i})=\underbrace{\lim }_{I} \underset{\overrightarrow{J^{\circ}}}{\lim } \operatorname{Hom}\left(Y_{j}, X_{i}\right)
$$

The category $\operatorname{Pro}(C)$ admits cofiltered projective limits, and if $C$ admits fiber products, then so is $\operatorname{Pro}(C)$. The canonical fully faithful functor $L: C \rightarrow \operatorname{Pro}(C)$ commutes with fiber products, but does not commute, in general, with cofiltered projective limits. One has $\underset{⿱}{\lim }{ }_{I} X_{i}={\underset{\leftarrow}{I}}_{I} L\left(X_{i}\right)$.

A pro- $k$-analytic space is an object of the category $\operatorname{Pro}(k-\mathcal{A} n)$. The category $\operatorname{Pro}(k-\mathcal{A} n)$ admits fiber products and cofiltered projective limits, and for a nonArchimedean field $K$ over $k$ there is the ground field extension functor $\operatorname{Pro}(k-\mathcal{A} n) \rightarrow \operatorname{Pro}(K-\mathcal{A} n): \mathbf{X}=\underbrace{}_{I} \lim _{I} X_{i} \mapsto \mathbf{X} \widehat{\otimes} K={\underset{\leftarrow}{I}}^{\lim }\left(X_{i} \widehat{\otimes} K\right)$. A pro-analytic space over $k$ is a pair $(K, \mathbf{X})$, where $K$ is a non-Archimedean field over $k$ and $\mathbf{X} \in \operatorname{Pro}(K-\mathcal{A} n)$. A morphism $(L, \mathbf{Y}) \rightarrow(K, \mathbf{X})$ is a pair consisting of an isometric embedding $K \hookrightarrow L$ and a morphism $\mathbf{Y} \rightarrow \mathbf{X} \widehat{\otimes}_{K} L$.

Let $\mathbf{X}=" \underset{\leftarrow}{I} " X_{i}$ be a pro- $k$-analytic space. It gives rise to a pro-object of the category of locally ringed spaces. Since the latter category admits cofiltered projective limits, we get the underlying locally ringed space $|\mathbf{X}|$ of $\mathbf{X}$. (We remark that the space $|\mathbf{X}|$ may be empty even when $\mathbf{X}$ is nontrivial.) If $x \in \mathbf{X}$ (i.e., $x \in|\mathbf{X}|$ ), then $\mathcal{O}_{\mathbf{X}, x}=\underset{\overrightarrow{I^{\circ}}}{\lim _{X_{i}}, x_{i}}$, where $x_{i}$ is the image of $x$ in $X_{i}$. The residue field of the local ring $\mathcal{O}_{\mathbf{X}, x}$ is denoted by $\kappa(x)$. Furthermore, let $\mathcal{H}(x)$ denote the completion of the field $\lim \mathcal{H}\left(x_{i}\right)$. If, for each $i$, the point $x_{i}$ has an affinoid neighborhood in $X_{i}$, then the field $\kappa(x)$ is quasicomplete and its completion coincides with $\mathcal{H}(x)$.

For a pro- $k$-analytic space $\mathbf{X}=\underset{I}{\lim } " X_{i}$ we define the category of "étale sheaves of sets" $\mathbf{T}(\mathbf{X})$ as the inductive $\operatorname{limit} \underset{\overrightarrow{I^{\circ}}}{\lim } \mathbf{T}\left(X_{i}\right)$ (see [SGA4], Exp. VI). Namely, objects of $\mathbf{T}(\mathbf{X})$ are pairs $(i, F)$, where $i \in I$ and $F \in \mathbf{T}\left(X_{i}\right)$. A representative of a morphism $(i, F) \rightarrow(j, G)$ is a triple $(\alpha, \beta, u)$, where $\alpha: l \rightarrow i$ and $\beta: l \rightarrow j$ are arrows in $I$ and $u: \nu_{\alpha}^{*}(F) \rightarrow \nu_{\beta}^{*}(G)$ is a morphism of sheaves on $X_{l}\left(\nu_{\alpha}\right.$ is the morphism $X_{l} \rightarrow X_{i}$ that corresponds to $\left.\alpha\right)$. Two representatives $(\alpha, \beta, u)$ and $\left(\alpha^{\prime}, \beta^{\prime}, u^{\prime}\right)$ of a morphism $(i, F) \rightarrow(j, G)$ are said to be equivalent if there exist arrows $\gamma: q \rightarrow l$ and $\gamma^{\prime}: q \rightarrow l^{\prime}$ such that $\alpha \circ \gamma=\alpha^{\prime} \circ \gamma^{\prime}, \beta \circ \gamma=\beta^{\prime} \circ \gamma^{\prime}$ and $\gamma^{*}(u)=\gamma^{\prime *}\left(u^{\prime}\right)$. A morphism is an equivalence class of representatives. We remark that if $\mathbf{X}=\underset{I}{\lim } \mathbf{X}_{i}$ is a cofiltered projective limit in the category $\operatorname{Pro}(k-\mathcal{A} n)$, then there is an equivalence of categories $\lim _{\overrightarrow{I^{\circ}}} \mathbf{T}\left(\mathbf{X}_{i}\right) \stackrel{\sim}{\longrightarrow} \mathbf{T}(\mathbf{X})$.

One also has the abelian categories of "abelian sheaves" $\mathbf{S}(\mathbf{X})$ and of "sheaves of $\Lambda$-modules" $\mathbf{S}(\mathbf{X}, \Lambda)$. There is a left exact functor $\mathbf{S}(\mathbf{X}) \rightarrow \mathcal{A} b: F \mapsto F(\mathbf{X})=$ $\lim _{\longrightarrow} F\left(X_{i}\right)$. Suppose that all of the morphisms $\nu_{\alpha}: X_{i} \rightarrow X_{j}$ are étale. Then the functors $\nu_{\alpha}^{*}: \mathbf{S}\left(X_{j}\right) \rightarrow \mathbf{S}\left(X_{i}\right)$ take injectives into injectives, and therefore the category $\mathbf{S}(\mathbf{X})$ has injectives. The values of the right derived functors of the functor $F \mapsto F(\mathbf{X})$ are $H^{q}(\mathbf{X}, F)=\underset{\overrightarrow{I^{\circ}}}{\lim _{i}} H^{q}\left(X_{i}, F\right)$. 
Let $\varphi: \mathbf{Y}=" \underbrace{\lim }_{J} " Y_{j} \rightarrow \mathbf{X}=\underbrace{}_{I} \lim " X_{i}$ be a morphism of pro-analytic spaces over $k$. Then one can define the inverse image functor $\varphi^{*}: \mathbf{T}(\mathbf{X}) \rightarrow \mathbf{T}(\mathbf{Y})$ and, in a situation we really need, the direct image functor $\varphi_{*}: \mathbf{T}(\mathbf{Y}) \rightarrow \mathbf{T}(\mathbf{X})$ as follows. If for each $i \in I$ we fix $\sigma(i) \in J$ and a morphism $\varphi_{i}: Y_{\sigma(i)} \rightarrow X_{i}$ determined by $\varphi$, then $\varphi^{*}(i, F)=\left(\sigma(i), \varphi_{i}^{*}(F)\right)$. Furthermore, suppose that there are a full cofinal subcategory $I^{\prime} \subset I$, a cofinal functor $\sigma: I^{\prime} \rightarrow J$, and a system of morphisms $\left\{\varphi_{i}: Y_{\sigma(i)} \rightarrow X_{i}\right\}_{i \in I^{\prime}}$ which defines $\varphi$ and such that for any arrow $\alpha: l \rightarrow i$ in $I^{\prime}$ the diagram

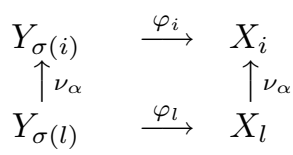

is commutative and cartesian. Suppose also that all of the morphisms $\nu_{\alpha}: X_{l} \rightarrow$ $X_{i}$ for arrows $\alpha$ in $I$ are étale. Then for any $F \in \mathbf{T}\left(Y_{\sigma(i)}\right)$ there is a canonical isomorphism $\nu_{\alpha}^{*} \varphi_{i_{*}}(F) \stackrel{\sim}{\longrightarrow} \varphi_{l_{*}} \nu_{\alpha}^{*}(F)$, and therefore the correspondence $(\sigma(i), F) \mapsto$ $\left(i, \varphi_{i_{*}}(F)\right), i \in I^{\prime}$, gives the required functor $\varphi_{*}$ which is right adjoint to $\varphi^{*}$. In this situation the category $\mathbf{S}(\mathbf{Y})$ has injectives, and there are the right derived functors $R^{q} \varphi_{*}: \mathbf{S}(\mathbf{Y}) \rightarrow \mathbf{S}(\mathbf{X})$.

For a $k$-analytic space $X$ we denote by $X-\mathcal{A} n$ the category of morphisms of $k$ analytic spaces $Y \rightarrow X$. Such an $Y$ is said to be an $X$-analytic space. If $\mathbf{X}=\underset{I}{=} \lim _{I} " X_{i}$ is a pro- $k$-analytic space, then an $\mathbf{X}$-analytic space is an object of the category $\mathbf{X}-\mathcal{A} n:=\lim _{\overrightarrow{I^{\circ}}} X_{i}-\mathcal{A} n$. If $P$ is a class of morphisms between $k$-analytic spaces which is preserved under any base change, then one can extend in the evident way the class $P$ to morphisms between $\mathbf{X}$-analytic spaces. Furthermore, if all of the morphisms $\nu_{\alpha}: X_{i} \rightarrow X_{j}$ are étale, then for any morphism of $\mathbf{X}$-analytic spaces $\varphi: \mathbf{Z} \rightarrow \mathbf{Y}$ the direct image functor $\varphi_{*}: \mathbf{S}(\mathbf{Z}) \rightarrow \mathbf{S}(\mathbf{Y})$ as well as the right derived functors $R^{q} \varphi_{*}$ are well defined.

Germs of analytic spaces (see [Ber2], §3.4) are examples of pro-analytic spaces, namely, there is a fully faithful functor

$$
k \text {-Germs } \rightarrow \operatorname{Pro}(k-\mathcal{A} n):(X, \Sigma) \mapsto X(\Sigma)=" \lim _{\mathcal{U} \supset \Sigma} " \mathcal{U}
$$

where $\mathcal{U}$ runs through open neighborhoods of $\Sigma$. The functor commutes with extensions of the ground field, but does not commute with fiber products. For example, let $\varphi: Y \rightarrow X$ be a morphism of $k$-analytic spaces and $x \in X$. Then the fiber product $Y \times_{X}(X, x)$ in the category $k$-Germs is the $k$-germ $\left(Y, \varphi^{-1}(x)\right)$, i.e., it gives rise to $Y\left(\varphi^{-1}(x)\right)=$ "lim" $\mathcal{V}$, where $\mathcal{V}$ runs through all open neighborhoods of the fiber $\varphi^{-1}(x)$. The corresponding fiber product $Y(x):=Y \times_{X} X(x)$ in the category $\operatorname{Pro}(k-\mathcal{A} n)$ is "lim" $\varphi^{-1}(\mathcal{U})$, where $\mathcal{U}$ runs through open neighborhoods of the point $x$. We remark that the canonical morphism $Y\left(\varphi^{-1}(x)\right) \rightarrow Y(x)$ induces an isomorphism between the underlying locally ringed spaces, and there is a morphism $Y_{x} \rightarrow Y\left(\varphi^{-1}(x)\right)$ which induces a homeomorphism between the underlying topological spaces. The space $Y(x)$ is an example of an $X(x)$-analytic space. And in fact any $X(x)$-analytic space $\mathbf{Y}$ is isomorphic to $Y(x)$ for some $Y \rightarrow X$. The fiber of $\mathbf{Y}$ over $x$ is, by definition, the $\mathcal{H}(x)$-analytic space $\mathbf{Y}_{x}:=Y_{x}$. 
For a $k$-germ $(X, \Sigma)$ there is an exact functor $\mathbf{T}(X(\Sigma)) \rightarrow \mathbf{T}(X, \Sigma): F \mapsto F_{(X, \Sigma)}$ which associates with a sheaf $F \in \mathbf{T}(\mathcal{U}), \mathcal{U} \supset \Sigma$, its pullback on $(X, \Sigma)$. The continuity theorem ([Ber2], 4.3.5) tells that if a $k$-germ $(X, \Sigma)$ is paracompact, then for all $F \in \mathbf{S}(X(\Sigma))$ and $q \geq 0$ there is a canonical isomorphism $H^{q}(X(\Sigma), F) \stackrel{\sim}{\longrightarrow}$ $H^{q}\left((X, \Sigma), F_{(X, \Sigma)}\right)$.

\section{§3. GAGA over the LOCAL RING OF A POINT}

Let $(S, s)$ be a $k$-germ such that $S$ is good at $s$, i.e., the point $s$ has an affinoid neighborhood in $S$, and let $A=\mathcal{O}_{S, s}$ and $\mathcal{S}=\operatorname{Spec}(A)$. We recall that $A=\lim \mathcal{A}_{W}$, where $W$ runs through affinoid neighborhoods of the point $s$, and that the rings $A$ and $\mathcal{A}_{W}$ are Noetherian. For an affinoid domain $V=\mathcal{M}\left(\mathcal{A}_{V}\right)$ we denote by $\mathcal{V}$ the affine scheme $\operatorname{Spec}\left(\mathcal{A}_{V}\right)$. By [EGA4], 8.8.2, for any scheme $\mathcal{X}$ of finite type over $\mathcal{S}$ there exist an affinoid neighborhood $V$ of $s$ and a scheme $\mathcal{X}_{V}$ of finite type over $\mathcal{V}$ such that $\mathcal{X} \stackrel{\sim}{\longrightarrow} \mathcal{X}_{V} \otimes_{\mathcal{V}} \mathcal{S}$ over $\mathcal{S}$. Furthermore, a projective limit of the projective system $\left\{\mathcal{X}_{W}=\mathcal{X}_{V} \otimes_{\mathcal{V}} \mathcal{W}\right\}_{V \supset W \ni s}$ exists in the category of schemes over $\mathcal{S}$, and one has $\mathcal{X} \stackrel{\sim}{\longrightarrow} \lim \mathcal{X}_{W}$. Finally, if $\mathcal{Y}$ is another scheme of finite type over $\mathcal{S}$ with $\mathcal{Y} \stackrel{\sim}{\longrightarrow} \mathcal{Y}_{V} \otimes \mathcal{V} \mathcal{S}$ for some scheme $\mathcal{Y}_{V}$ of finite type over $\mathcal{V}$, then there is a canonical bijection $\lim \operatorname{Hom}_{\mathcal{W}}\left(\mathcal{Y}_{W}, \mathcal{X}_{W}\right) \stackrel{\sim}{\longrightarrow} \operatorname{Hom}_{\mathcal{S}}(\mathcal{Y}, \mathcal{X})$.

By $\left[\right.$ Ber2],$\S 2.6$, one can associate with the scheme $\mathcal{X}_{V}$ a $k$-analytic space $\mathcal{X}_{V}^{\mathrm{an}}=$ $\left(\mathcal{X}_{V}\right)^{\text {an }}$ closed over $V$. The $V$-analytic space $\mathcal{X}_{V}^{\text {an }}$ gives rise to an $S(s)$-analytic space $\mathcal{X}_{V}^{\mathrm{an}}(s)$. From the above EGA-facts it follows that $\mathcal{X}_{V}^{\mathrm{an}}(s)$ does not depend on the choice of $V$ and $\mathcal{X}_{V}$ up to a canonical isomorphism, and therefore we can set $\mathcal{X}^{\text {an }}=\mathcal{X}_{V}^{\text {an }}(s)$. (For example, $\mathcal{S}^{\text {an }}=S(s)$.) It follows also that the correspondence $\mathcal{X} \mapsto \mathcal{X}^{\text {an }}$ is a functor which commutes with fiber products. Moreover, if $\left(S^{\prime}, s^{\prime}\right) \rightarrow$ $(S, s)$ is a morphism of germs over $k$, then there is a canonical isomorphism

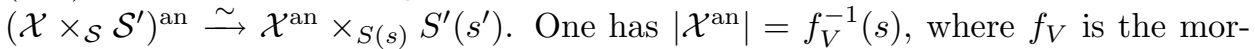
phism $\mathcal{X}_{V}^{\mathrm{an}} \rightarrow V$, and, for $x \in \mathcal{X}^{\mathrm{an}}, \mathcal{O}_{\mathcal{X}^{\mathrm{an}}, x}=\mathcal{O}_{\mathcal{X}_{V}^{\mathrm{an}}, x}$. From [Ber2], 2.6.2, it follows that the canonical morphism of locally ringed spaces $\pi: \mathcal{X}^{\text {an }} \rightarrow \mathcal{X}$ is flat and its image coincides with the closed fiber of $\mathcal{X}, \mathcal{X}_{s}=\mathcal{X} \otimes_{A} \kappa(s)$. For a point $x \in \mathcal{X}^{\text {an }}$ (i.e., $x \in\left|\mathcal{X}^{\text {an }}\right|$ ) we denote by $\mathbf{x}$ its image in $\mathcal{X}$.

By the analytification of the closed fiber we mean the $\mathcal{H}(s)$-analytic space $\mathcal{X}_{s}^{\text {an }}=$ $\left(\mathcal{X}^{\mathrm{an}}\right)_{s}=\left(\mathcal{X}_{s} \otimes_{\kappa(s)} \mathcal{H}(s)\right)^{\text {an }}$ (and not the $S(s)$-analytic space $\left.\left(\mathcal{X}_{s}\right)^{\text {an }}\right)$. The canonical morphism $\mathcal{X}_{s}^{\text {an }} \rightarrow \mathcal{X}^{\text {an }}$ induces a homeomorphism between the underlying topological spaces. Similarly, by the analytification of the geometric closed fiber of $f, \mathcal{X}_{\bar{s}}=$ $\mathcal{X} \otimes_{A} \kappa(s)^{\text {a }}$, we mean the $\widehat{\mathcal{H}(s)^{\mathrm{a}}}$-analytic space $\mathcal{X}_{\bar{s}}^{\text {an }}=\left(\mathcal{X}^{\text {an }}\right)_{\bar{s}}=\left(\mathcal{X}_{\bar{s}} \otimes_{\kappa(s)^{\mathrm{s}}} \widehat{\mathcal{H}(s)^{\mathrm{a}}}\right)^{\text {an }}$. There are the evident functors $\mathbf{T}\left(\mathcal{X}_{s}\right) \rightarrow \mathbf{T}\left(\mathcal{X}_{s}^{\text {an }}\right)$ and $\mathbf{T}\left(\mathcal{X}_{\bar{s}}\right) \rightarrow \mathbf{T}\left(\mathcal{X}_{\bar{s}}^{\text {an }}\right): \mathcal{F} \mapsto \mathcal{F}^{\text {an }}$. We remark that since all of the schemes $\mathcal{X}_{W}$ are quasicompact and quasiseparated, then the isomorphism $\mathcal{X} \stackrel{\sim}{\longrightarrow} \lim \mathcal{X}_{W}$ induces an equivalence of categories $\underset{\lim }{\longrightarrow} \mathbf{T}\left(\mathcal{X}_{W}\right) \stackrel{\sim}{\longrightarrow} \mathbf{T}(\mathcal{X})$. In this way one gets a functor $\mathbf{T}(\mathcal{X}) \rightarrow \mathbf{T}\left(\mathcal{X}^{\mathrm{an}}\right): \mathcal{F} \mapsto \mathcal{F}^{\mathrm{an}}$.

3.1. Proposition. Let $\varphi: \mathcal{Y} \rightarrow \mathcal{X}$ be a morphism of schemes of finite type over $\mathcal{S}$. Then

(i) $\varphi$ is separated (proper, finite, quasifinite, a closed immersion) if and only if $\varphi^{\text {an }}$ possesses the same property;

(ii) $\varphi$ is flat quasifinite (unramified, étale, smooth) if and only if $\varphi^{\text {an }}$ possesses the same property. 
Proof. (i) follows from [EGA4], 8.10.5, and [Ber2], 2.6.9, 3.1.7.

(ii) is proved in the same way as the corresponding statements [Ber2], 3.2.10, 3.3.11 and 3.5.8, using the following fact which is an easy consequence of [Ber2], 2.6.10.

3.2. Lemma. Suppose that $\varphi$ is finite. Let $\mathbf{y} \in \mathcal{Y}$ and $x \in \mathcal{X}^{\text {an }}$ be points with $\varphi(\mathbf{y})=\mathbf{x}$, and let $\varphi^{\mathrm{an}^{-1}}(x)=\left\{y_{1}, \ldots, y_{n}\right\}$ and $\varphi^{\mathrm{an}^{-1}}(x) \cap \pi^{-1}(\mathbf{y})=\left\{y_{1}, \ldots, y_{m}\right\}$, $m \leq n$. Then there is an isomorphism of rings

$$
\mathcal{O}_{\mathcal{X}^{\mathrm{an}, x}} \otimes_{\mathcal{O}_{\mathcal{X}, \mathbf{x}}} \mathcal{O}_{\mathcal{Y}, \mathbf{y}} \stackrel{\sim}{\longrightarrow} \prod_{i=1}^{m} \mathcal{O}_{\mathcal{Y}^{\mathrm{an}}, y_{i}} \times \prod_{i=m+1}^{n}\left(\mathcal{O}_{\mathcal{Y}^{\mathrm{an}}, y_{i}}\right)_{\mathbf{m}_{\mathbf{y}}}
$$

where $\left(\mathcal{O}_{y^{a n}, y_{i}}\right)_{\mathbf{m}_{\mathbf{y}}}$ is the localization with respect to the complement of the maximal ideal $\mathbf{m}_{\mathbf{y}}$ of $\mathcal{O}_{\mathcal{Y}, \mathbf{y}}$.

The following is a direct consequence of the comparison theorem for cohomology with compact support [Ber2], ( $\$ 7.1)$.

3.3. Proposition. Let $\varphi: \mathcal{Y} \rightarrow \mathcal{X}$ be a proper morphism between schemes of finite type over $\mathcal{S}$, and let $\mathcal{F}$ be an abelian torsion sheaf on $\mathcal{Y}$. Then for any $q \geq 0$ there is a canonical isomorphism

$$
\left(R^{q} \varphi_{*} \mathcal{F}\right)^{\text {an }} \stackrel{\sim}{\longrightarrow} R^{q} \varphi_{*}^{\text {an }}\left(\mathcal{F}^{\text {an }}\right) .
$$

The following fact is an essential ingredient in the proof of the comparison theorem for vanishing cycles 6.1 .

3.4. Theorem. Let $x$ be a point of $\mathcal{X}^{\text {an }}$ and $\mathbf{x}$ its image in $\mathcal{X}$. Then the field $\kappa(x)$ is separable over $k(\mathbf{x})$.

Recall that an extension of fields $L / K$ is called separable if the tensor product $L \otimes_{K} K^{p^{-1}}$ is a field, where $p=\operatorname{char}(K)$. In this case the tensor product $L \otimes_{K} K^{p^{-\infty}}$ is also a field, and $L^{\mathrm{s}}$ is separable over $K^{\mathrm{s}}$. Therefore the tensor product $L^{\mathrm{s}} \otimes_{K^{\mathrm{s}}} K^{\mathrm{a}}$ is a field and, in particular, if $K^{\prime}$ is a finite extension of $K^{\mathrm{s}}$, then $\left[L^{\mathrm{s}} K^{\prime}: L^{\mathrm{s}}\right]=$ $\left[K^{\prime}: K^{\mathrm{s}}\right]$.

First of all we want to show that the theorem follows from the following fact.

3.5. Proposition. Suppose that $\mathcal{O}_{S, s}$ is a field, i.e., $\mathcal{O}_{S, s}=\kappa(s)$. If $\mathcal{X}$ is reduced at $\mathbf{x}$, then $\mathcal{X}^{\text {an }}$ is reduced at $x$.

Indeed, to prove the theorem, it suffices to assume that $\mathcal{O}_{S, s}=\kappa(s), \mathcal{X}$ is reduced and irreducible, and $\mathbf{x}$ is the generic point of $\mathcal{X}$. Let $K$ be a finite purely inseparable extension of the field $k(\mathbf{x})$. Then we can shrink $\mathcal{X}$ and find a finite radicial morphism $\varphi: \mathcal{Y} \rightarrow \mathcal{X}$ such that $\mathcal{Y}$ is reduced and, for the generic point $\mathbf{y}$ of $\mathcal{Y}$, one has $k(\mathbf{y})=K$. By Proposition 3.5, the $S(s)$-analytic space $\mathcal{Y}^{\text {an }}$ is also reduced. Since the morphism $\varphi$ is radicial, there is a unique point $y \in \mathcal{Y}^{\text {an }}$ whose images in $\mathcal{Y}$ and $\mathcal{X}^{\text {an }}$ are $\mathbf{y}$ and $x$, respectively. By Lemma 3.2, we get $k(\mathbf{y}) \otimes_{k(\mathbf{x})} \kappa(x) \stackrel{\sim}{\longrightarrow} \mathcal{O}_{y^{\text {an }}, y}$. Since the local ring $\mathcal{O}_{\mathcal{y}^{\text {an }}, y}$ is reduced and finite over the field $\kappa(x)$, it follows that that it is a field (i.e., it coincides with $\kappa(y)$ ), and the theorem follows.

To prove Proposition 3.5, we need the following two lemmas (one does not assume in them that $\mathcal{O}_{S, s}$ is a field). Let $\mathcal{X}_{0}$ denote the set of closed points of the closed fiber of $\mathcal{X}$. Furthermore, for an $S(s)$-analytic space $\mathbf{X}$ let $\mathbf{X}_{0}$ denote the set of points $x \in \mathbf{X}$ with $[\kappa(x): \kappa(s)]<\infty$. We set $\mathcal{X}_{0}^{\text {an }}=\left(\mathcal{X}^{\text {an }}\right)_{0}$. 
3.6. Lemma. The map $\mathcal{X}^{\text {an }} \rightarrow \mathcal{X}$ induces a bijection $\mathcal{X}_{0}^{\text {an }} \stackrel{\sim}{\rightarrow} \mathcal{X}_{0}$. Furthermore, if $x \in \mathcal{X}_{0}^{\text {an }}$, then there is an isomorphism of completions $\widehat{\mathcal{O}}_{\mathcal{X}, \mathbf{x}} \stackrel{\sim}{\longrightarrow} \widehat{\mathcal{O}}_{\mathcal{X}}$ an,$x$.

Proof. Let $\mathbf{x} \in \mathcal{X}_{0}$. For $n \geq 1$ we set $\mathcal{Y}=\operatorname{Spec}\left(\mathcal{O}_{\mathcal{X}, \mathbf{x}} / \mathbf{m}_{\mathbf{x}}^{n}\right)$. The scheme $\mathcal{Y}$ consists of one point $\mathbf{y}$ and is finite over $\mathcal{S}$. Therefore $\mathcal{Y}^{\text {an }}$ consists of one point $y$ and, by Lemma 3.2, one has $\mathcal{O}_{\mathcal{Y}, \mathbf{y}}=\mathcal{O}_{\mathcal{X}, \mathbf{x}} / \mathbf{m}_{\mathbf{x}}^{n} \stackrel{\sim}{\longrightarrow} \mathcal{O}_{\mathcal{Y}^{\text {an }}, y}$. Furthermore, there is a canonical closed immersion $\mathcal{Y} \rightarrow \mathcal{X}$ that takes $\mathbf{y}$ to $\mathbf{x}$. From Lemma 3.2 it follows that $\mathcal{Y}^{\text {an }} \rightarrow$ $\mathcal{X}^{\text {an }}$ is also a closed immersion, and the point $x$ is the only preimage of $\mathbf{x}$ in $\mathcal{X}^{\text {an }}$. (In particular, $\mathcal{X}_{0}^{\text {an }} \stackrel{\sim}{\longrightarrow} \mathcal{X}_{0}$.) Moreover, one has $\mathcal{O}_{\mathcal{Y}^{\mathrm{an}}, y}=\mathcal{O}_{\mathcal{X}^{\mathrm{an}}, x} / \mathbf{m}_{\mathbf{x}}^{n} \mathcal{O}_{\mathcal{X}^{\mathrm{an}}, x}$. It follows that $\mathcal{O}_{\mathcal{X}, \mathbf{x}} / \mathbf{m}_{\mathbf{x}}^{n} \stackrel{\sim}{\longrightarrow} \mathcal{O}_{\mathcal{Y}}$ an,$y$. If $n=1$ we get $\mathbf{m}_{x}=\mathbf{m}_{\mathbf{x}} \mathcal{O}_{\mathcal{X}}$ an,$x$ and $k(\mathbf{x}) \stackrel{\sim}{\longrightarrow} \kappa(x)$.

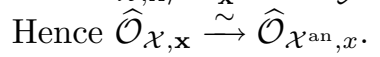

3.7. Lemma. Suppose that the valuation on $\kappa(s)$ is nontrivial. Then for any $S(s)$ analytic space $\mathbf{X}$ closed over $S(s)$ the set $\mathbf{X}_{0}$ is everywhere dense in $\mathbf{X}$.

Proof. For a closed morphism $f: X \rightarrow S$, we set

$$
X(s)_{0}=\left\{x \in f^{-1}(s) \mid[\kappa(x): \kappa(s)]<\infty\right\}
$$

and

$$
\left(X_{s}\right)_{0}=\left\{x \in X_{s} \mid[\mathcal{H}(x): \mathcal{H}(s)]<\infty\right\} .
$$

(Note that there is a homeomorphism $X_{s} \stackrel{\sim}{\longrightarrow} f^{-1}(s)$.) First, we claim that if $f$ is the projection $X=S \times E^{d} \rightarrow S$, where $E^{d}$ is the closed unit polydisc in $\mathbf{A}^{d}$ with center at zero, then $X(s)_{0}$ is everywhere dense in $f^{-1}(s)$. Indeed, one has

$$
X(s)_{0}=\left\{\alpha \in \kappa(s)^{\mathrm{a}}|| \alpha \mid \leq 1\right\}^{d} / G
$$

and

$$
\left(X_{s}\right)_{0}=\left\{\alpha \in \mathcal{H}(s)^{\mathrm{a}}|| \alpha \mid \leq 1\right\}^{d} / G,
$$

where $G=G\left(\kappa(s)^{\mathrm{s}} / \kappa(s)\right)=G\left(\mathcal{H}(s)^{\mathrm{s}} / \mathcal{H}(s)\right)$. Since the field $\kappa(s)^{\mathrm{s}}$ is everywhere dense in $\mathcal{H}(s)^{\mathrm{a}}$ and the set $X(s)_{0}$ is everywhere dense in $f^{-1}(s)$, the claim follows.

Now let $f: X \rightarrow S$ be a closed morphism and $x \in f^{-1}(s)$. We have to show that, for any affinoid neighborhood $V=\mathcal{M}(\mathcal{B})$ of $x$, there exists a point $x^{\prime} \in V$ over $s$ with $\left[\kappa\left(x^{\prime}\right): \kappa(s)\right]<\infty$. Shrinking $V$, we may assume that $\operatorname{dim}_{x}\left(V_{s}\right)=\operatorname{dim}\left(V_{s}\right)$. We may also assume that $S=\mathcal{M}(\mathcal{A})$ is $k$-affinoid. Since $x \in \operatorname{Int}(V / S)$, there is an admissible epimorphism $\mathcal{A}\left\{r_{1}^{-1} T_{1}, \ldots, r_{n}^{-1} T_{n}\right\} \rightarrow \mathcal{B}$ with $\left|f_{i}(X)\right|<r_{i}, 1 \leq i \leq n$, where $f_{i}$ is the image of $T_{i}$ in $\mathcal{B}$. Replacing $V$ by the Weierstrass domain $V\left(r_{i}^{\prime-1} f_{i}\right)$ for some $\left|f_{i}(x)\right|<r_{i}^{\prime}<r_{i}$ with $r_{i}^{\prime} \in \sqrt{\left|\mathcal{H}(s)^{*}\right|}$, we may assume that the $\mathcal{H}(s)$ affinoid algebra $\mathcal{B} \widehat{\otimes}_{\mathcal{A}} \mathcal{H}(s)$ is strictly $\mathcal{H}(s)$-affinoid. By the Noether normalization lemma, we can find elements $g_{1}, \ldots, g_{d} \in \mathcal{B} \widehat{\otimes}_{\mathcal{A}} \mathcal{H}(s)$ such that there is an admissible, injective and finite homomorphism $\mathcal{H}(s)\left\{T_{1}, \ldots, T_{d}\right\} \rightarrow \mathcal{B}: T_{i} \mapsto g_{i}$. It defines a finite surjective morphism $V_{s} \rightarrow E_{\mathcal{H}(s)}^{d}$. (One has $d=\operatorname{dim}\left(V_{s}\right)$.) Since the image of $\mathcal{B} \otimes_{\mathcal{A}} \kappa(s)$ in $\mathcal{B} \widehat{\otimes}_{\mathcal{A}} \mathcal{H}(s)$ is everywhere dense, we may assume that $g_{1}, \ldots, g_{d}$ come from $\mathcal{B} \otimes_{\mathcal{A}} \kappa(s)$. Shrinking $S$, we may assume that they come from $\mathcal{B}$. Furthermore, consider the morphism $g: V \rightarrow S \times \mathbf{A}^{d}$ defined by the functions $g_{1}, \ldots, g_{d}$. Since $x \in \operatorname{Int}(V / S)$, it follows that $g$ is finite at $x$ ([Ber2], 3.1.4). Let $W$ be the preimage of $S \times E^{d}$ in $V$. The induced morphism $h: W \rightarrow S \times E^{d}$ is also finite at $x$. This means that there are open neighborhoods $\mathcal{W}$ of $x$ in $W$ and $\mathcal{U}$ of $h(x)$ in $S \times E^{d}$ such that $h$ induces a finite morphism $\mathcal{W} \rightarrow \mathcal{U}$. Since $\operatorname{dim}_{x}\left(\mathcal{W}_{s}\right)=\operatorname{dim}\left(\mathcal{W}_{s}\right)=d$, from [Ber1], 3.2.4, it follows that we can shrink $\mathcal{U}$ and assume that the morphism $\mathcal{W}_{s} \rightarrow \mathcal{U}_{s}$ is surjective. We can find a point $y^{\prime} \in \mathcal{U}$ over $s$ with $\left[\kappa\left(y^{\prime}\right): \kappa(s)\right]<\infty$. If $x^{\prime}$ is a point of $\mathcal{W}$ over $y^{\prime}$, then $\left[\kappa\left(x^{\prime}\right): \kappa(s)\right]<\infty$. 
Proof of Proposition 3.5. We can shrink $\mathcal{X}$ and assume that it is reduced. Let $V$ be an affinoid neighborhood of $s$ such that $\mathcal{X}$ comes from a scheme $\mathcal{X}_{V}$ of finite type over $\mathcal{V}$, and let $Y$ be the set of points $y \in \mathcal{X}_{V}^{\mathrm{an}}$ such that $\mathcal{X}_{V}^{\mathrm{an}}$ is not reduced at $y$. By [Ber2], 2.2.1, the set $Y$ is Zariski closed in $\mathcal{X}_{V}^{\text {an }}$, and therefore it can be considered as a $k$-analytic space closed over $V$. It gives rise to an $S(s)$-analytic space $\mathbf{Y}$ closed over $S(s)$. Assume that $\mathbf{Y}$ is nonempty. We claim that then the set $\mathbf{Y}_{0}$ is nonempty. Indeed, if the valuation on $\kappa(s)$ is nontrivial, this follows from Lemma 3.7. Suppose that the valuation on $\kappa(s)$ is trivial. The closed fiber $\mathcal{X}_{s}$ is an $\mathcal{H}(s)$-analytic space, and $\mathbf{Y}_{s}$ is a Zariski closed subset of $\mathcal{X}_{s}^{\text {an }}$. By [Ber1], §3.5, one has $\mathbf{Y}_{s}=\mathcal{Z}^{\text {an }}$, where $\mathcal{Z}$ is a Zariski closed subset of $\mathcal{X}_{s}$. It follows that there exists a point $\mathbf{y} \in \mathcal{Z}$ with $[k(\mathbf{y}): \kappa(s)]<\infty$, and therefore $\mathbf{Y}_{0}$ is nonempty. Now let $y \in \mathbf{Y}_{0}$. By Lemma 3.6, one has $\widehat{\mathcal{O}}_{\mathcal{X}, \mathbf{y}} \stackrel{\sim}{\longrightarrow} \widehat{\mathcal{O}}_{\mathcal{X}}^{\text {an }, y}$. Since $\mathcal{O}_{S, s}=\kappa(s)$, it follows that $\mathcal{O}_{\mathcal{X}, \mathbf{y}}$ is a localization of a ring which is finitely generated over the field $\kappa(s)$. Such a ring is reduced if and only if its completion is reduced (see [EGA4], 7.8.3). It follows that $\mathcal{O}_{\mathcal{X}^{\text {an }}, y}$ is reduced, which is impossible because $y \in \mathbf{Y}$.

3.8. Remark. One can construct the functor $\mathcal{X} \mapsto \mathcal{X}^{\text {an }}$ in another way so that the construction works also over $\mathbf{C}$ and $\mathbf{R}$. Namely, one has $A=\lim A_{i}$, where $A_{i}$ runs through subrings of $A$ which are finitely generated over $k$. For any scheme $\mathcal{X}$ of finite type over $\mathcal{S}$ there exist $i$ and a scheme $\mathcal{X}_{i}$ of finite type over $\mathcal{S}_{i}=\operatorname{Spec}\left(A_{i}\right)$ such that $\mathcal{X} \stackrel{\sim}{\longrightarrow} \mathcal{X}_{i} \otimes_{\mathcal{S}_{i}} \mathcal{S}$ over $\mathcal{S}$. By GAGA over the field $k$, one can associate with $\mathcal{S}_{i}$ and $\mathcal{X}_{i} k$-analytic spaces $S_{i}=\mathcal{S}_{i}^{\text {an }}$ and $X_{i}=\mathcal{X}_{i}^{\text {an }}$. The canonical homomorphism $A_{i} \rightarrow A=\mathcal{O}_{S, s}$ defines a point $s_{i} \in S_{i}$ and a morphism of $k$ germs $(S, s) \rightarrow\left(S_{i}, s_{i}\right)$. The latter is induced by a morphism $\mathcal{U} \rightarrow S_{i}$ from an open neighborhood $\mathcal{U}$ of the point $s$. One has $\mathcal{X}^{\text {an }}=\left(X_{i} \times_{S_{i}} \mathcal{U}\right)(s)$. The essential difference of the Archimedean situation from the non-Archimedean one is that in this situation the map $\left|\mathcal{X}^{\text {an }}\right| \rightarrow$ $\mathcal{X}_{s}$ is injective and its image coincides with the set of closed points of the closed fiber $\mathcal{X}_{s}$. (In particular, for a point $x \in \mathcal{X}^{\text {an }}$, one has $\kappa(x)=k(\mathbf{x})$.)

\section{$\S 4$. The VANishing CYCles FUNCTOR}

Beginning with this section, we assume that, for the $k$-germ $(S, s)$ from $\S 3$, $A=\mathcal{O}_{S, s}$ is a discrete valuation ring. In this case the scheme $\mathcal{S}=\operatorname{Spec}(A)$ consists of the closed point $s=\operatorname{Spec}(\kappa(s))$ and the generic point $\eta=\operatorname{Spec}(\mathcal{K})$, where $\mathcal{K}$ is the fraction field of $A$. We denote by $\mathbf{S}$ the pro- $k$-analytic space $\mathcal{S}^{\text {an }}=S(s)$. The scheme $\eta$ is of finite type over $\mathcal{S}$, and therefore one can associate with it a pro- $k$-analytic space $\eta^{\text {an }}$. One has $\eta^{\text {an }}=" \lim "(\mathcal{U} \backslash T)$, where $\mathcal{U}$ runs through open neighborhoods of $s$ and $T \subset S$ is a Zariski closed subset that goes through the point $s$. For an $\mathbf{S}$-analytic space $\mathbf{X}$ we set $\mathbf{X}_{\eta}=\mathbf{X} \times \mathbf{s} \eta^{\text {an }}$. (For example, $\mathbf{S}_{\eta}=\eta^{\text {an }}$.)

Since $\mathcal{K}$ is the fraction field of the Henselian discrete valuation ring $A$, its valuation extends uniquely to a valuation on the separable closure $\mathcal{K}^{\mathrm{s}}$, and the integral closure $\bar{A}$ of $A$ in $\mathcal{K}^{\mathrm{s}}$ is a local ring. The residue field $\widetilde{\mathcal{K}^{\mathrm{s}}}$ is an algebraic closure $\kappa(s)^{\mathrm{a}}$ of $\kappa(s)$. Let $\overline{\mathcal{S}}=\operatorname{Spec}(\bar{A}), \bar{\eta}=\operatorname{Spec}\left(\mathcal{K}^{\mathrm{s}}\right)$ and $\bar{s}=\operatorname{Spec}\left(\kappa(s)^{\mathrm{a}}\right)$, and let $\nu$ be the canonical homomorphism $G_{\eta}=G\left(\mathcal{K}^{\mathrm{s}} / \mathcal{K}\right) \rightarrow G_{s}=G\left(\kappa(s)^{\mathrm{s}} / \kappa(s)\right)$. Recall the definition of the vanishing cycles functor $\Psi_{\eta}: \mathbf{T}\left(\mathcal{X}_{\eta}\right) \rightarrow \mathbf{T}_{G_{\eta}}\left(\mathcal{X}_{\bar{s}}\right)$ for a scheme $\mathcal{X}$ over $\mathcal{S}$.

One sets $\overline{\mathcal{X}}=\mathcal{X} \times_{\mathcal{S}} \overline{\mathcal{S}}, \mathcal{X}_{\bar{\eta}}=\mathcal{X} \times_{\mathcal{S}} \bar{\eta}$ and $\mathcal{X}_{\bar{s}}=\mathcal{X} \times_{\mathcal{S}} \bar{s}$. Furthermore, for a finite extension $\mathcal{L}$ of $\mathcal{K}$ in $\mathcal{K}^{\mathrm{s}}$, one denotes by $A_{\mathcal{L}}$ the integral closure of $A$ in $\mathcal{L}$ and set $\mathcal{S}_{\mathcal{L}}=\operatorname{Spec}\left(A_{\mathcal{L}}\right)$. The scheme $\mathcal{S}_{\mathcal{L}}$ consists of the closed point $s_{\mathcal{L}}=\operatorname{Spec}(\widetilde{\mathcal{L}})$ and 
the generic point $\eta_{\mathcal{L}}=\operatorname{Spec}(\mathcal{L})$. One sets $\mathcal{X}_{\mathcal{L}}=\mathcal{X} \times_{\mathcal{S}} \mathcal{S}_{\mathcal{L}}, \mathcal{X}_{\eta_{\mathcal{L}}}=\mathcal{X} \times_{\mathcal{S}} \eta_{\mathcal{L}}$ and $\mathcal{X}_{s_{\mathcal{L}}}=\mathcal{X} \times s_{\mathcal{S}} s_{\mathcal{L}}$. There is a commutative diagram

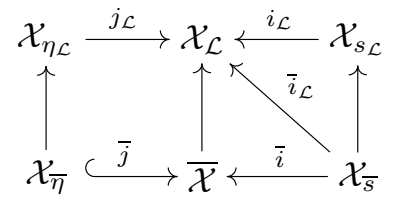

For $\mathcal{F} \in \mathbf{T}\left(\mathbf{X}_{\eta}\right)$, let $\mathcal{F}_{\mathcal{L}}$ and $\overline{\mathcal{F}}$ denote the pullbacks of $\mathcal{F}$ on $\mathcal{X}_{\eta_{\mathcal{L}}}$ and $\mathcal{X}_{\bar{\eta}}$. Then

$$
\Psi_{\eta}(\mathcal{F})=\underset{\lim }{\longrightarrow} \bar{i}_{\mathcal{L}}^{*} j_{\mathcal{L} *}\left(\mathcal{F}_{\mathcal{L}}\right) \stackrel{\sim}{\longrightarrow} \bar{i}^{*} \bar{j}_{*}(\overline{\mathcal{F}}),
$$

where $\mathcal{L}$ runs through finite extensions of $\mathcal{K}$ in $\mathcal{K}^{\mathrm{s}}$.

We now return to analytic geometry. For a finite extension $\mathcal{L}$ of $\mathcal{K}$ in $\mathcal{K}^{\mathrm{s}}$, we set $\mathbf{S}_{\mathcal{L}}=\mathcal{S}_{\mathcal{L}}^{\text {an }}$ (this space consists of one point $s_{\mathcal{L}}$ ) and $\overline{\mathbf{S}}=" \lim " \mathbf{S}_{\mathcal{L}}$. We also set $\mathbf{S}_{\eta_{\mathcal{L}}}=\left(\eta_{\mathcal{L}}\right)^{\text {an }}$ and $\mathbf{S}_{\bar{\eta}}="{ }^{\prime i m} " \mathbf{S}_{\eta_{\mathcal{L}}}$. Furthermore, for an $\mathbf{S}$-analytic space $\mathbf{X}$, we set $\mathbf{X}_{\mathcal{L}}=\mathbf{X} \times_{\mathbf{S}} \mathbf{S}_{\mathcal{L}}, \mathbf{X}_{\eta_{\mathcal{L}}}=\mathbf{X} \times_{\mathbf{S}} \mathbf{S}_{\eta_{\mathcal{L}}}, \overline{\mathbf{X}}=\mathbf{X} \times_{\mathbf{S}} \overline{\mathbf{S}}$, and $\mathbf{X}_{\bar{\eta}}=\mathbf{X} \times_{\mathbf{S}} \mathbf{S}_{\bar{\eta}}$. There is a commutative diagram of morphisms of pro-analytic spaces

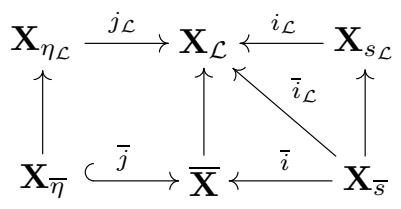

For $F \in \mathbf{T}\left(\mathbf{X}_{\eta}\right)$, we denote by $F_{\mathcal{L}}$ the pullback of $F$ on $\mathbf{X}_{\eta_{\mathcal{L}}}$ and define the vanishing cycles functor $\Psi_{\eta}: \mathbf{T}\left(\mathbf{X}_{\eta}\right) \rightarrow \mathbf{T}_{G_{\eta}}\left(\mathbf{X}_{\bar{s}}\right)$ by

$$
\Psi_{\eta}(F)=\underline{\lim } \bar{i}_{\mathcal{L}}^{*} j_{\mathcal{L}_{*}}\left(F_{\mathcal{L}}\right)
$$

where $\mathcal{L}$ runs through finite extensions of $\mathcal{K}$ in $\mathcal{K}^{\mathrm{s}}$. It follows from the definition that, for a scheme $\mathcal{X}$ of finite type over $\mathcal{S}$ and a sheaf of sets (resp. abelian groups) $\mathcal{F}$ on $\mathcal{X}_{\eta}$, there is a canonical morphism of sheaves $\mathcal{X}_{\bar{s}}^{\text {an }},\left(\Psi_{\eta}(\mathcal{F})\right)^{\text {an }} \rightarrow \Psi_{\eta}\left(\mathcal{F}^{\text {an }}\right)$ (resp. $\left.\left(R^{q} \Psi_{\eta}(\mathcal{F})\right)^{\text {an }} \rightarrow R^{q} \Psi_{\eta}\left(\mathcal{F}^{\text {an }}\right), q \geq 0\right)$.

For a morphism of $\mathbf{S}$-analytic spaces $\varphi: \mathbf{Y} \rightarrow \mathbf{X}$ we denote by $\varphi_{s}, \varphi_{\bar{s}}, \varphi_{\eta}$ and $\varphi_{\bar{\eta}}$ the induced morphisms $\mathbf{Y}_{s} \rightarrow \mathbf{X}_{s}, \mathbf{Y}_{\bar{s}} \rightarrow \mathbf{X}_{\bar{s}}, \mathbf{Y}_{\eta} \rightarrow \mathbf{X}_{\eta}$ and $\mathbf{Y}_{\bar{\eta}} \rightarrow \mathbf{X}_{\bar{\eta}}$, respectively. The following statements follow straightforwardly from the weak base change theorem 5.3.6 and the smooth base change theorem 7.8.1 from [Ber2].

4.1. Proposition. Let $\varphi: \mathbf{Y} \rightarrow \mathbf{X}$ be a morphism of $\mathbf{S}$-analytic spaces.

(i) If $\varphi$ is compact, then for any $F^{*} \in D^{+}\left(\mathbf{Y}_{\eta}\right)$ there is a canonical isomorphism $R \Psi_{\eta}\left(R \varphi_{\eta_{*}} F^{\cdot}\right) \stackrel{\sim}{\longrightarrow} R \varphi_{\bar{s}_{*}}\left(R \Psi_{\eta} F^{\cdot}\right)$. In particular, if $\mathbf{X}$ is an $\mathbf{S}$-analytic space compact over $\mathbf{S}$, then for any abelian sheaf $F$ on $\mathbf{X}_{\eta}$ there is a spectral sequence

$$
E_{2}^{p, q}=H^{p}\left(\mathbf{X}_{\bar{s}}, R^{q} \Psi_{\eta}(F)\right) \Longrightarrow H^{p+q}\left(\mathbf{X}_{\bar{\eta}}, F\right)
$$

(ii) If $\varphi$ is smooth, then for any abelian torsion sheaf on $\mathbf{X}_{\eta}$ with torsion orders prime to $\operatorname{char}(\widetilde{k})$ and any $q \geq 0$ there is a canonical isomorphism

$$
\varphi_{\bar{s}}^{*}\left(R^{q} \Psi_{\eta}(F)\right) \stackrel{\sim}{\longrightarrow} R^{q} \Psi_{\eta}\left(\varphi_{\eta}^{*} F\right) .
$$


Let $\mathcal{L}$ be a Galois extension of $\mathcal{K}$ in $\mathcal{K}^{\mathrm{s}}$ that contains $\mathcal{K}^{\mathrm{nr}}$, the maximal unramified extension of $\mathcal{K}$. The residue field $\widetilde{\mathcal{L}}$ is an algebraic extension of $\kappa(s)$ that contains $\kappa(s)^{\mathrm{s}}$, and therefore its completion coincides with $\widehat{\mathcal{H}(s)^{\mathrm{a}}}$. One has, for an $\mathbf{S}$-analytic space $\mathbf{X}$, a left exact functor $\Psi_{\eta, \mathcal{L}}: \mathbf{T}\left(\mathbf{X}_{\eta}\right) \rightarrow \mathbf{T}_{G(\mathcal{L} / \mathcal{K})}\left(\mathbf{X}_{\bar{s}}\right)$ defined by $\Psi_{\eta, \mathcal{L}}(F)=$ $\stackrel{\lim }{{ }_{i}^{*}}{ }_{\mathcal{N}} j_{\mathcal{N} *}\left(F_{\mathcal{N}}\right)$, where $\mathcal{N}$ runs through finite extensions of $\mathcal{K}$ in $\mathcal{L}$. For example, if $\mathcal{L}=\mathcal{K}^{\mathrm{nr}}$, then $R^{q} \Psi_{\eta, \mathcal{K}^{\mathrm{nr}}}(F)=\bar{i}^{*}\left(R^{q} j_{*}(F)\right)$, where $\bar{i}=\bar{i}_{\mathcal{K}}: \mathbf{X}_{\bar{s}} \rightarrow \mathbf{X}$ and $j=j_{\mathcal{K}}: \mathbf{X}_{\eta} \rightarrow \mathbf{X}$. One has the following simple fact.

4.2. Proposition. In the above situation, for any $F \in \mathbf{S}\left(\mathbf{X}_{\eta}\right)$ there is a spectral sequence

$$
E_{2}^{p, q}=\mathcal{H}^{p}\left(G\left(\mathcal{K}^{\mathrm{s}} / \mathcal{L}\right), R^{q} \Psi_{\eta}(F)\right) \Longrightarrow R^{p+q} \Psi_{\eta, \mathcal{L}}(F)
$$

Let $S^{\prime} \rightarrow S$ be a morphism of good $k$-analytic spaces, and let $s \in S$ and $s^{\prime} \in S^{\prime}$ be points such that $\mathcal{O}_{S, s}$ and $\mathcal{O}_{S^{\prime}, s^{\prime}}$ are discrete valuation rings, the image of $s^{\prime}$ in $S$ is $s$, and the induced homomorphism $\mathcal{O}_{S, s} \rightarrow \mathcal{O}_{S^{\prime}, s^{\prime}}$ is injective. One has morphisms of affine schemes $\mathcal{S}^{\prime}=\operatorname{Spec}\left(\mathcal{O}_{S^{\prime}, s^{\prime}}\right) \rightarrow \mathcal{S}=\operatorname{Spec}\left(\mathcal{O}_{S, s}\right)$ and of pro- $k$-analytic spaces $\mathbf{S}^{\prime}=S^{\prime}\left(s^{\prime}\right) \rightarrow \mathbf{S}=S(s)$. Furthermore, let $X \rightarrow S^{\prime}$ be a morphism of $k$-analytic spaces. It gives rise to an $\mathbf{S}^{\prime}$-analytic space $\mathbf{X}^{\prime}$ (with $\mathbf{X}_{s^{\prime}}^{\prime}=X_{s^{\prime}}$ ). The induced morphism $X \rightarrow S$ gives rise to an $\mathbf{S}$-analytic space $\mathbf{X}$ (with $\mathbf{X}_{s}=X_{s}$ ). Thus, there is a commutative diagram of morphisms of pro- $k$-analytic spaces

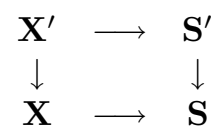

The morphism $\mathbf{X}^{\prime} \rightarrow \mathbf{X}$ induces the evident inverse image functor $\mathbf{T}\left(\mathbf{X}_{\eta}\right) \rightarrow$ $\mathbf{T}\left(\mathbf{X}_{\eta^{\prime}}^{\prime}\right): F \mapsto F^{\prime}$. Let $\mathcal{K}$ and $\mathcal{K}^{\prime}$ be the fraction fields of $\mathcal{O}_{S, s}$ and $\mathcal{O}_{S^{\prime}, s^{\prime}}$, respectively. We fix an embedding of fields $\mathcal{K}^{\mathrm{s}} \hookrightarrow \mathcal{K}^{\prime \mathrm{s}}$ over the canonical embedding $\mathcal{K} \hookrightarrow \mathcal{K}^{\prime}$. It induces a homomorphism of Galois groups $G_{\eta^{\prime}} \rightarrow G_{\eta}$. It induces also an embedding $\kappa(s)^{\mathrm{a}} \hookrightarrow \kappa\left(s^{\prime}\right)^{\mathrm{a}}$ and, therefore, a morphism $\lambda: \mathbf{X}_{\bar{s}^{\prime}}^{\prime} \rightarrow \mathbf{X}_{\bar{s}}$ of analytic spaces over $k$.

4.3. Proposition. In the above situation, assume that the morphism $S^{\prime} \rightarrow S$ is smooth, and let $\mathcal{L}=\mathcal{K}^{\prime n r} \mathcal{K}^{\mathrm{s}}$. Then for any $F \in \mathbf{S}\left(\mathbf{X}_{\eta}\right)$ and any $q \geq 0$ there is a canonical isomorphism $\lambda^{*}\left(R^{q} \Psi_{\eta}(F)\right) \stackrel{\sim}{\longrightarrow} R^{q} \Psi_{\eta^{\prime}, \mathcal{L}}\left(F^{\prime}\right)$ compatible with the action of Galois groups.

Proof. Let $\mathcal{N}$ be a finite Galois extension of $\mathcal{K}$ in $\mathcal{K}^{\mathrm{s}}$ and $\mathcal{N}^{\prime}=\mathcal{K}^{\prime} \mathcal{N}$. We can shrink $S$ and assume that $\mathbf{S}_{\eta}$ comes from $S \backslash T$, where $T$ is a Zariski closed subset of $S$, the morphism $\mathbf{S}_{\mathcal{N}} \rightarrow \mathbf{S}$ comes from a flat finite morphism $S_{\mathcal{N}} \rightarrow S$, and the sheaf $F$ comes from $X \backslash Y$, where $Y$ is the preimage of $T$ in $X$. Furthermore, we can shrink $S^{\prime}$ and assume that the morphism $\mathbf{S}_{\mathcal{N}^{\prime}}^{\prime} \rightarrow \mathbf{S}^{\prime}$ comes from a flat finite morphism $S_{\mathcal{N}^{\prime}}^{\prime} \rightarrow S^{\prime}$. We may assume also that $S$ and $S_{\mathcal{N}}$ are regular. By hypothesis, the morphism $S^{\prime} \times_{S} S_{\mathcal{N}} \rightarrow S_{\mathcal{N}}$ is smooth, and therefore $S^{\prime} \times_{S} S_{\mathcal{N}}$ is regular. It follows that the ring $\mathcal{O}_{S^{\prime}, s^{\prime}} \otimes_{\mathcal{O}_{S, s}} \mathcal{O}_{S_{\mathcal{N}}, s_{\mathcal{N}}}$ is the integral closure of $\mathcal{O}_{S^{\prime}, s^{\prime}}$ in $\mathcal{K}^{\prime} \otimes_{\mathcal{K}} \mathcal{N}$. The latter is a direct product of finite separable extensions of $\mathcal{K}^{\prime}$. One of these factors is $\mathcal{N}^{\prime}$. We get a point $t \in S^{\prime} \times_{S} S_{\mathcal{N}}$ over $s^{\prime}$ with the local ring $\mathcal{O}_{S_{\mathcal{N}^{\prime}}^{\prime}, s_{\mathcal{N}^{\prime}}^{\prime}}$, and therefore we can shrink $S^{\prime}$ and find a morphism $S_{\mathcal{N}^{\prime}}^{\prime} \rightarrow S^{\prime} \times{ }_{S} S_{\mathcal{N}}$ over $S^{\prime}$ such that it takes the point $s_{\mathcal{N}^{\prime}}^{\prime}$ to $t$ and is a local isomorphism at $s_{\mathcal{N}^{\prime}}^{\prime}$. We get a morphism

$$
\lambda_{\mathcal{N}}: X_{\mathcal{N}^{\prime}}^{\prime}=X \times_{S^{\prime}} S_{\mathcal{N}^{\prime}}^{\prime} \rightarrow X \times_{S^{\prime}}\left(S^{\prime} \times_{S} S_{\mathcal{N}}\right)=X_{\mathcal{N}}
$$


that induces an open immersion of the preimage of an open neighborhood of the point $s_{\mathcal{N}^{\prime}}^{\prime}$ in $X_{\mathcal{N}^{\prime}}^{\prime}$ to $X_{\mathcal{N}}$. This implies that there is an isomorphism of sheaves

$$
\lambda_{s_{\mathcal{N}}}^{*}\left(i_{\mathcal{N}}^{*} j_{\mathcal{N} *}(F)\right) \stackrel{\sim}{\longrightarrow} i_{\mathcal{N}^{\prime}}^{\prime}{ }^{*} j_{\mathcal{N}^{\prime} *}^{\prime}\left(F^{\prime}\right),
$$

where $X_{\mathcal{N}} \backslash Y_{\mathcal{N}} \stackrel{j_{\mathcal{N}}}{\hookrightarrow} X_{\mathcal{N}} \stackrel{i_{\mathcal{N}}}{\longleftarrow} X_{s_{\mathcal{N}}}, X_{\mathcal{N}^{\prime}}^{\prime} \backslash Y_{\mathcal{N}^{\prime}}^{\prime} \stackrel{j_{\mathcal{N}}^{\prime}}{\hookrightarrow} X_{\mathcal{N}^{\prime}}^{\prime} \stackrel{i_{\mathcal{N}^{\prime}}^{\prime}}{\longleftarrow} X_{s_{\mathcal{N}^{\prime}}^{\prime}}^{\prime}, Y_{\mathcal{N}}$ and $Y_{\mathcal{N}^{\prime}}^{\prime}$ are the preimages of $T$ in $X_{\mathcal{N}}$ and $X_{\mathcal{N}^{\prime}}^{\prime}$, and $\lambda_{s_{\mathcal{N}}}$ is the morphism $X_{s_{\mathcal{N}^{\prime}}^{\prime}}^{\prime} \rightarrow X_{s_{\mathcal{N}}}$. The proposition follows.

4.4. Corollary. The Galois group $P=G\left(\mathcal{K}^{\prime \mathrm{s}} / \mathcal{K}^{\prime \mathrm{nr}} \mathcal{K}^{\mathrm{s}}\right)$ is a pro-p-group, where $p=\operatorname{char}(k)$, and if $F$ is torsion with torsion orders prime to $p$, then there is a canonical isomorphism $\lambda^{*}\left(R^{q} \Psi_{\eta}(F)\right) \stackrel{\sim}{\longrightarrow} R^{q} \Psi_{\eta^{\prime}}\left(F^{\prime}\right)^{P}$.

Proof. To prove the first statement, it suffices to verify that the field $\mathcal{K}^{\text {nr }} \mathcal{K}^{\mathrm{s}}$ contains $\mathcal{K}^{\prime \text { mr }}$, the maximal moderately ramified extension of $\mathcal{K}^{\prime}$. For this it suffices to show that $\mathcal{K}^{\prime \mathrm{mr}}=\mathcal{K}^{\prime \mathrm{nr}} \mathcal{K}^{\mathrm{mr}}$, or, equivalently, that the homomorphism of Galois groups $G\left(\mathcal{K}^{\prime \mathrm{mr}} / \mathcal{K}^{\prime \mathrm{nr}}\right) \rightarrow G\left(\mathcal{K}^{\mathrm{mr}} / \mathcal{K}^{\mathrm{nr}}\right)$ is injective. By [Ber2], 2.4.4, the first (resp. second) group is canonically isomorphic to $\operatorname{Hom}\left(\sqrt{\left|\mathcal{K}^{*}\right|} /\left|\mathcal{K}^{*}\right|, \widetilde{\mathcal{K}}^{s^{*}}\right)$ (resp. $\left.\operatorname{Hom}\left(\sqrt{\left|\mathcal{K}^{\prime *}\right|} /\left|\mathcal{K}^{\prime *}\right|, \widetilde{\mathcal{K}}^{s^{*}}\right)\right)$. Since $\left|\mathcal{K}^{*}\right|$ is a subgroup of finite index in the cyclic group $\left|\mathcal{K}^{\prime *}\right|$, then $\sqrt{\left|\mathcal{K}^{*}\right|}=\sqrt{\left|\mathcal{K}^{\prime *}\right|}$, and the required fact follows. The second statement is an easy consequence of the first one and Propositions 4.2 and 4.3.

\section{$\S 5$. Smooth analytic SPaCES}

Let $(S, s)$ be a $k$-germ such that $s$ is contained in the interior $\operatorname{Int}(S)$ of $S$ (in particular, $S$ is good at $s$ ) and $\mathcal{O}_{S, s}$ is a discrete valuation ring.

5.1. Theorem. Suppose that the field $k$ is perfect, and let $n$ be an integer prime to char $(k)$. Then for $\mathbf{X}=\mathbf{S}$ one has $\Psi_{\eta}(\mathbf{Z} / n \mathbf{Z})_{\eta}=(\mathbf{Z} / n \mathbf{Z})_{\bar{s}}$ and $R^{q} \Psi_{\eta}(\mathbf{Z} / n \mathbf{Z})_{\eta}=0$ for $q \geq 1$.

To prove the theorem, we show that the assumptions guarantee the smoothness of the $k$-analytic spaces $S_{\mathcal{L}}$ and $T_{\mathcal{L}}$ at the point $s_{\mathcal{L}}$, and, after that, we apply the cohomological purity theorems from [Ber2] and [Ber4].

5.2. Theorem. Let $X$ be a $k$-analytic space and $K$ a perfect non-Archimedean field over $k$. Then, for a point $x \in X$, the following are equivalent:

(a) $X$ is smooth at $x$;

(b) $x \in \operatorname{Int}(X)$ and there exists an open neighborhood $\mathcal{U}$ of $x$ such that the $K$ analytic space $\mathcal{U} \widehat{\otimes} K$ is regular;

(c) $x \in \operatorname{Int}(X)$ and the $K$-analytic space $X \widehat{\otimes} K$ is regular at some point over $x$.

Proof. The implications (a) $\Longrightarrow(\mathrm{b}) \Longrightarrow(\mathrm{c})$ are trivial. To prove the implication (c) $\Longrightarrow$ (a), we need the following fact.

5.3. Lemma. The following properties of a $k$-analytic space $X$ at a point $x \in X$ with $d=\operatorname{dim}_{x}(X)$ are equivalent:

(a) $X$ is smooth at $x$;

(b) $x \in \operatorname{Int}(X)$ and $\Omega_{X, x}$ is a free $\mathcal{O}_{X, x}$-module of rank $d$;

(c) $x \in \operatorname{Int}(X)$ and $\Omega_{X, x}$ is generated over $\mathcal{O}_{X, x}$ by at most $d$ elements. 
Proof. The implication (a) $\Longrightarrow$ (b) follows from [Ber2], 3.5.4. The implication (b) $\Longrightarrow(\mathrm{c})$ is trivial. Suppose that (c) is true. Shrinking $X$, we may assume that $X$ is closed and there are functions $f_{1}, \ldots, f_{d} \in \mathcal{O}(X)$ such that the sheaf $\Omega_{X}$ is generated by $d f_{1}, \ldots, d f_{d}$. We claim that the morphism $f: X \rightarrow Y=\mathbf{A}^{d}$, defined by $f_{1}, \ldots, f_{d}$, is étale. Indeed, the exact sequence $f^{*}\left(\Omega_{Y}\right) \longrightarrow \Omega_{X} \longrightarrow \Omega_{X / Y} \longrightarrow 0$ (see [Ber2], 3.3.2(i)) implies that $\Omega_{X / Y}=0$. It follows that $f$ has discrete fibers. Since $f$ is closed, $f$ is quasifinite, by [Ber2], 3.1.4, and therefore $f$ is unramified. We have to verify that $f$ is flat. Let $y=f(y)$. Since $\mathcal{O}_{X, x}$ is a finite unramified $\mathcal{O}_{Y, y}$-algebra, and the ring $\mathcal{O}_{Y, y}$ is normal, by [SGA1], Exp. I, 9.5(ii), it suffices to show that the canonical homomorphism $\mathcal{O}_{Y, y} \rightarrow \mathcal{O}_{X, x}$ is injective. But this is clear because $\operatorname{dim}_{y}(Y)=d=\operatorname{dim}_{x}(X)$.

5.4. Corollary. Let $x$ be an inner point of a $k$-analytic space $X$ such that the local ring $\mathcal{O}_{X, x}$ is regular and $\mathcal{H}(x)=k^{\prime}$ or $k^{\prime} \hat{\otimes} K_{r}$, where $k^{\prime}$ is a finite separable extension of $k$ and $r \notin \sqrt{\left|k^{*}\right|}$. Then $X$ is smooth at $x$.

Proof. Suppose first that $\mathcal{H}(x)=k^{\prime}$. Then the set $\{x\}$ is Zariski closed in $X$. By [Ber2], 3.3.2(ii), there is an exact sequence $\mathbf{m}_{x} / \mathbf{m}_{x}^{2} \longrightarrow \Omega_{X} \otimes_{\mathcal{O}_{X}} k^{\prime} \longrightarrow \Omega_{k^{\prime} / k} \longrightarrow$ 0 . It follows that $\Omega_{X, x}$ is generated over $\mathcal{O}_{X, x}$ by at most $\operatorname{dim}_{k^{\prime}}\left(\mathbf{m}_{x} / \mathbf{m}_{x}^{2}\right)=$ $\operatorname{dim}\left(\mathcal{O}_{X, x}\right)=\operatorname{dim}_{x}(X)$ elements. Suppose now that $\mathcal{H}(x)=k^{\prime} \widehat{\otimes} K_{r}$. We can replace $k$ by $k^{\prime}$ and assume that $\mathcal{H}(x)=K_{r}$. We may assume also that $X$ is $k$ affinoid. Consider the canonical morphism $X^{\prime}=X \widehat{\otimes} K_{r} \rightarrow X$. From [Ber2], 2.2.1 and 2.2.5, it follows that there exists a point $x^{\prime} \in \pi^{-1}(x)$ which is contained in the Zariski open set $\mathcal{V}$ of regular points of $X^{\prime}$. Furthermore, the fiber $\pi^{-1}(x)$ is isomorphic to the annulus $A(r, r)_{K_{r}} \subset \mathbf{A}_{K_{r}}^{1}$. It follows that there exists a point $x^{\prime \prime} \in \mathcal{V} \cap \pi^{-1}(x)$ for which $\mathcal{H}\left(x^{\prime \prime}\right)$ is a finite separable extension of $K_{r}$. By the first case, $\Omega_{X^{\prime}, x^{\prime \prime}}$ is generated over $\mathcal{O}_{X^{\prime}, x^{\prime \prime}}$ by at $\operatorname{most} \operatorname{dim}_{x^{\prime \prime}}\left(X^{\prime}\right)$ elements. Since $\Omega_{X^{\prime}, x^{\prime \prime}}=\Omega_{X, x} \otimes_{\mathcal{O}_{X, x}} \mathcal{O}_{X^{\prime}, x^{\prime \prime}}$, it follows that $\Omega_{X, x}$ is generated over $\mathcal{O}_{X, x}$ by at $\operatorname{most}_{\operatorname{dim}_{x^{\prime \prime}}}\left(X^{\prime}\right) \leq \operatorname{dim}_{x}(X)$ elements.

Suppose that $x \in \operatorname{Int}(X)$ and some point $x^{\prime} \in X \widehat{\otimes} K$ over $x$ is regular. By Lemma 5.3 , it suffices to verify that $\Omega_{X, x}$ is generated over $\mathcal{O}_{X, x}$ by at most $\operatorname{dim}_{x}(X)$ elements. Since $\Omega_{X^{\prime}, x^{\prime}}=\Omega_{X, x} \otimes_{\mathcal{O}_{X, x}} \mathcal{O}_{X^{\prime}, x^{\prime}}$, it suffices to verify that $\Omega_{X^{\prime}, x^{\prime}}$ is generated over $\mathcal{O}_{X^{\prime}, x^{\prime}}$ by at most $\operatorname{dim}_{x^{\prime}}\left(X^{\prime}\right) \leq \operatorname{dim}_{x}(X)$ elements. The situation is reduced to the following. Suppose that the field $k$ is perfect. Then, for any point $x \in \operatorname{Int}(X)$ such that the local ring $\mathcal{O}_{X, x}$ is regular, $\Omega_{X, x}$ is generated over $\mathcal{O}_{X, x}$ by at most $d=\operatorname{dim}_{x}(X)$ elements. For this we need the following fact.

5.5. Lemma. Suppose that $k$ is perfect, and let $X$ be a closed $k$-analytic space. Then the set of points $x \in X$ with $\mathcal{H}(x)=k^{\prime}$ or $k^{\prime} \widehat{\otimes} K_{r}$ (as in Corollary 5.4) is everywhere dense in $X$.

Proof. If the valuation on $k$ is nontrivial, then the the space $X$ is strictly $k$-analytic (see [Ber1], 3.1.2), and therefore the set $X_{0}=\{x \in X \mid[\mathcal{H}(x): k]<\infty\}$ is everywhere dense in $X$. Suppose that the valuation on $k$ is trivial. It suffices to show that any affinoid neighborhood $U$ of a point $x \in X$ contains a point $y$ with $\mathcal{H}(y)=k^{\prime}$ or $k^{\prime} \widehat{\otimes} K_{r}$, where $k^{\prime}$ is a finite extension of $k$ and $0<r<1$. For this we take a closed immersion $U \rightarrow E\left(0 ; r_{1}, \ldots, r_{n}\right) \subset \mathbf{A}^{n}$ with $\left|T_{i}(x)\right|<r_{i}$. Furthermore, we take a number $0<r<1$ with $\left|T_{i}(x)\right|<r^{l_{i}}<r_{i}$ for some integers $l_{i}$, and set $V=U \cap E\left(0 ; r^{l_{1}}, \ldots, r^{l_{n}}\right)$. Then $V^{\prime}=V \widehat{\otimes} K_{r}$ is a strictly $K_{r}$-affinoid space, and therefore the set $V_{0}^{\prime}$ is everywhere dense in $V^{\prime}$. Let $y$ be the image in $V$ of some 
point $y^{\prime} \in V_{0}^{\prime}$. We claim that the field $\mathcal{H}(y)$ is of the required form. Suppose that $\mathcal{H}(y)$ is infinite over $k$. One has $k \subset \mathcal{H}(y) \subset \mathcal{H}\left(y^{\prime}\right)$ and $\left[\mathcal{H}\left(y^{\prime}\right): K_{r}\right]<\infty$. Since $\widetilde{K}_{r}=k, k$ is perfect, and the ring $\mathcal{H}(y)^{\circ}$ is Henselian, there exists a finite extension $k^{\prime}$ of $k$ in $\mathcal{H}(y)$ with $k^{\prime} \stackrel{\sim}{\longrightarrow} \widetilde{\mathcal{H}(y)}$. Furthermore, the group $\left|\mathcal{H}(y)^{*}\right|$ is generated by a number $0<r^{\prime}<1$. Let $t$ be an element of $\mathcal{H}(y)$ with $|t|=r^{\prime}$. Then the series $\sum_{i=-\infty}^{\infty} a_{i} t^{i}, a_{i} \in k^{\prime}$, are convergent in $\mathcal{H}(y)$, and any element of $\mathcal{H}(y)$ can be represented in a unique way as such a series. It follows that $\mathcal{H}(y)=k^{\prime} \widehat{\otimes} K_{r^{\prime}}$.

Shrinking $X$, we may assume that $X$ is closed, regular, and of pure dimension $d$. Let $Y$ be the set of points $x \in X$ such that $\Omega_{X, x}$ is generated over $\mathcal{O}_{X, x}$ by at least $d+1$ elements, and assume that $Y$ is nonempty. Then $Y$ is a Zariski closed subset of $X$, and therefore it can be considered as a closed $k$-analytic space. By Lemma 5.5 , there exists a point $y \in Y$ with $\mathcal{H}(y)=k^{\prime}$ or $k^{\prime} \widehat{\otimes} K_{r}$. By Corollary 5.4, $\Omega_{X, y}$ is generated over $\mathcal{O}_{X, y}$ by at most $d$ elements. The latter is impossible, and therefore Theorem 5.2 is proved.

5.6. Corollary. The set of smooth points in a closed $k$-analytic space $X$ is Zariski open. Furthermore, if the field $k$ is perfect, this set coincides with $\operatorname{Reg}(X)$, the set of regular points of $X$.

Proof of Theorem 5.1. We can shrink $S$ and assume that $\mathbf{S}_{\eta}$ comes from $S \backslash T$, where $T$ is a Zariski closed subset of $S$, and that $S$ and $T$ are smooth. Furthermore, for a fixed finite separable extension $\mathcal{L}$ of $\mathcal{K}$, we can shrink $S$ and assume that the morphism $\mathbf{S}_{\mathcal{L}} \rightarrow \mathbf{S}$ comes from a flat finite morphism $S_{\mathcal{L}} \rightarrow S$. Since $S_{\mathcal{L}}$ is regular at the point $s_{\mathcal{L}}$, from Theorem 5.2 it follows that we can shrink $S$ and assume that $S_{\mathcal{L}}$ and $T_{\mathcal{L}}$, the preimage of $T$ in $S_{\mathcal{L}}$, are smooth. By the cohomological purity theorem ([Ber2], 7.4.5, and [Ber4], 2.1), applied to the smooth pair of codimension one $S_{\mathcal{L}} \backslash T_{\mathcal{L}} \stackrel{j_{\mathcal{L}}}{\hookrightarrow} S_{\mathcal{L}} \stackrel{i_{\mathcal{L}}}{\longleftarrow} T_{\mathcal{L}}$, one has $j_{\mathcal{L} *}(\mathbf{Z} / n \mathbf{Z})_{S_{\mathcal{L}} \backslash T_{\mathcal{L}}}=(\mathbf{Z} / n \mathbf{Z})_{S_{\mathcal{L}}}, R^{1} j_{\mathcal{L} *}\left(\mu_{n, S_{\mathcal{L}} \backslash T_{\mathcal{L}}}\right) \stackrel{\sim}{\longrightarrow}$ $i_{\mathcal{L} *}(\mathbf{Z} / n \mathbf{Z})_{T_{\mathcal{L}}}$ and $R^{q} j_{\mathcal{L} *}(\mathbf{Z} / n \mathbf{Z})_{S_{\mathcal{L}} \backslash T_{\mathcal{L}}}=0$ for $q \geq 2$. Theorem 5.1 easily follows from this.

5.7. Corollary. Suppose that the field $k$ is perfect, and let $\mathbf{X}$ be an $\mathbf{S}$-analytic space smooth over $\mathbf{S}$. Then, for any finite locally constant abelian sheaf $F$ on $\mathbf{X}$ with torsion orders prime to $\operatorname{char}(k)$, one has $\Psi_{\eta}\left(F_{\eta}\right)=F_{\bar{s}}$ and $R^{q} \Psi_{\eta}\left(F_{\eta}\right)=0$ for $q \geq 1$.

\section{§6. THE COMPARISON THEOREM FOR VANISHING CYCLES}

In this section we assume that the field $k$ is perfect and $(S, s)$ is a $k$-germ such that $s \in \operatorname{Int}(S)$ and $A=\mathcal{O}_{S, s}$ is a discrete valuation ring.

6.1. Theorem. Let $\mathcal{X}$ be a scheme of finite type over $\mathcal{S}=\operatorname{Spec}(A)$, and let $\mathcal{F}$ be an abelian constructible sheaf on $\mathcal{X}_{\eta}$ with torsion orders prime to $\operatorname{char}(k)$. Then for any $q \geq 0$ there is a canonical isomorphism

$$
\left(R^{q} \Psi_{\eta}(\mathcal{F})\right)^{\text {an }} \stackrel{\sim}{\longrightarrow} R^{q} \Psi_{\eta}\left(\mathcal{F}^{\text {an }}\right) .
$$

6.2. Remark. Recall that, by Deligne's theorem 3.2 from [SGA $\left.4 \frac{1}{2}\right]$, Th. Finitude, the sheaves $R^{q} \Psi_{\eta}(\mathcal{F})$ are constructible. The proof of Theorem 6.1 uses the induction reasoning from the proof of Deligne's theorem and does not work in the classical situation over $\mathbf{C}$. But in the case covered by the corresponding theorem 
from [SGA7], Exp. XIV, the statement is easily deduced as follows from the comparison theorem for étale cohomology ([Ber2], 7.5.1, [Ber4], 3.1). (Moreover, in this case one does not need the assumption that the field $k$ is perfect.) Namely, this is the case when $\mathcal{X}=\mathcal{Y} \times_{\mathcal{R}} \mathcal{S}$, where $\mathcal{R}$ is an algebraic curve over $k, \mathcal{Y}$ is a scheme of finite type over $\mathcal{R}$, and $\mathcal{S} \rightarrow \mathcal{R}$ is a morphism which induces an isomorphism of $k$-germs $(S, s) \stackrel{\sim}{\longrightarrow}\left(\mathcal{R}^{\text {an }}, s\right)$, and $\mathcal{F}$ comes from a constructible sheaf $\mathcal{G}$ on $\mathcal{Y}_{\eta}$, where $\mathcal{Y}_{\eta}$ is the preimage of $\mathcal{R} \backslash\{s\}$ in $\mathcal{Y}$. We may assume that $\mathcal{R}$ is regular and connected. Let $K$ be the field of rational functions on $\mathcal{R}$, and fix an embedding $K^{\mathrm{s}} \hookrightarrow \mathcal{K}^{\mathrm{s}}$. Since $K$ is everywhere dense in $\mathcal{K}$ and $\mathcal{K}$ is quasicomplete, one has $\mathcal{K}^{\mathrm{s}}=K^{\mathrm{s}} \mathcal{K}$. For a finite extension $L$ of $K$, let $\mathcal{R}_{L}$ denote the normalization of $\mathcal{R}$ in $L$, and let $\mathcal{Y}_{L}=\mathcal{Y} \times_{\mathcal{R}} \mathcal{R}_{L}$. The embedding $L \hookrightarrow \mathcal{K}^{\mathrm{s}}$ defines a point $s_{L} \in \mathcal{R}_{L}$. There are morphisms $\mathcal{Y}_{\eta_{L}}=\mathcal{Y}_{L} \backslash\left(\mathcal{Y}_{L}\right)_{s_{L}} \stackrel{j_{L}}{\hookrightarrow} \mathcal{Y}_{L} \stackrel{\bar{i}_{L}}{\longleftarrow} \mathcal{Y}_{\bar{s}}$, and one has $R^{q} \Psi_{\eta}(\mathcal{F})=\lim _{\longrightarrow} \bar{i}_{L}^{*}\left(R^{q} j_{L *} \mathcal{G}\right)$, where $L$ runs through finite extensions of $K$ in $K^{\mathrm{s}}$. On the other hand, since $\mathcal{K}^{\mathrm{s}}=K^{\mathrm{s}} \mathcal{K}$, one has

$$
R^{q} \Psi_{\eta}\left(\mathcal{F}^{\mathrm{an}}\right)=\lim _{\underline{i}} \bar{i}_{L}^{\mathrm{an} *}\left(R^{q} j_{L *}^{\mathrm{an}} \mathcal{G}^{\mathrm{an}}\right) .
$$

The comparison theorem for étale cohomology implies that

$$
\left(R^{q} \Psi_{\eta}(\mathcal{F})\right)^{\text {an }} \stackrel{\sim}{\longrightarrow} R^{q} \Psi_{\eta}\left(\mathcal{F}^{\text {an }}\right) .
$$

Proof of Theorem 6.1. First of all we remark that it suffices to assume that $\mathcal{X}_{\eta}$ is everywhere dense in $\mathcal{X}$. We prove the theorem by induction on $d=\operatorname{dim}\left(\mathcal{X}_{\eta}\right)$.

Step 1. The theorem is true for $d=0$.

We may assume that $\mathcal{X}$ is reduced. Using Proposition 4.1(i), we can replace $\mathcal{X}$ by its normalization, and therefore we may assume that $\mathcal{X}$ is the normalization of $\mathcal{S}$ in a finite extension $\mathcal{N}$ of $\mathcal{K}$. Since $\mathcal{F}$ has a resolution $0 \rightarrow \mathcal{F} \rightarrow \mathcal{F}^{0} \rightarrow \mathcal{F}^{1} \rightarrow \ldots$ with $\mathcal{F}^{i}$ of the form $\varphi_{\eta_{*}}(\mathbf{Z} / n \mathbf{Z})_{\mathcal{X}_{\eta}^{\prime}}$, where $\varphi: \mathcal{X}^{\prime} \rightarrow \mathcal{X}$ is the normalization of $\mathcal{X}$ in a finite extension of $\mathcal{N}$, we again can apply Proposition 4.1(i) and reduce the situation to the case $\mathcal{F}=\Lambda_{\mathcal{X}_{\eta}}$, where $\Lambda=\mathbf{Z} / n \mathbf{Z}$. Furthermore, since the scheme $\mathcal{X}_{\bar{s}}$ is zero-dimensional, it suffices to verify that $H^{0}\left(\mathcal{X}_{\bar{s}}, R^{q} \Psi_{\eta}\left(\Lambda_{\mathcal{X}_{\eta}}\right)\right) \stackrel{\sim}{\longrightarrow}$ $H^{0}\left(\mathcal{X}_{\bar{s}}^{\text {an }}, R^{q} \Psi_{\eta}\left(\Lambda_{\mathcal{X}_{\eta}^{\text {an }}}\right)\right)$ or, equivalently, that $H^{q}\left(\mathcal{X}_{\bar{\eta}}, \Lambda\right) \stackrel{\sim}{\longrightarrow} H^{q}\left(\mathcal{X}_{\bar{\eta}}^{\text {an }}, \Lambda\right)$. For the latter, we may assume that $\mathcal{N}$ is separable over $\mathcal{K}$, but then it suffices to consider the case $\mathcal{N}=\mathcal{K}$, i.e., $\mathcal{X}=\mathcal{S}$. In this case $\Psi_{\eta}\left(\Lambda_{\eta}\right)=\Lambda_{\bar{s}}$ and $R^{q} \Psi_{\eta}\left(\Lambda_{\eta}\right)=0$ for $q>0$ and, by Theorem 5.1, the similar facts are true for $\mathbf{S}$.

Suppose now that $d \geq 1$ and the theorem is true for the schemes whose generic fiber has dimension at most $d-1$.

Step 2. The homomorphism $\left(R^{q} \Psi_{\eta}(\mathcal{F})\right)^{\text {an }} \rightarrow R^{q} \Psi_{\eta}\left(\mathcal{F}^{\text {an }}\right)$ is an isomorphism at any point of $\mathcal{X}_{\bar{s}}^{\mathrm{an}}$ whose image in the scheme $\mathcal{X}_{s}$ is not a closed point.

Let $y \in \mathcal{X}_{\bar{s}}^{\text {an }}$ be such a point, and let $x$ and $\mathbf{x}$ be its images in $\mathcal{X}_{s}^{\text {an }}$ and $\mathcal{X}_{s}$, respectively. Since our statement is local, we may assume that $\mathcal{X}$ is affine and, moreover, that $\mathcal{X}$ is a closed subscheme of the affine space $A_{\mathcal{S}}^{m}$. By hypothesis, there exists a projection $\varphi: \mathcal{X} \rightarrow A_{\mathcal{S}}^{1}$ such that $\mathbf{s}^{\prime}=\varphi(\mathbf{x})$ is the generic point of the closed fiber of $A_{\mathcal{S}}^{1}$. Then we may shrink $\mathcal{X}$ and assume that the generic fiber of $\varphi$ has dimension $d-1$. Let $s^{\prime}$ be the image of $x$ in the closed fiber of $\mathbf{A}_{S}^{1}=\left(A_{\mathcal{S}}^{1}\right)^{\text {an }}$, i.e., $s^{\prime}=\varphi^{\text {an }}(x)$, and let $\left(S^{\prime}, s^{\prime}\right)$ be the $k$-germ $\left(\mathbf{A}_{S}^{1}, s^{\prime}\right)$. To prove the statement, it suffices to show that the inverse images of the sheaves $R^{q} \Psi_{\eta}(\mathcal{F})^{\text {an }}$ and $R^{q} \Psi_{\eta}\left(\mathcal{F}^{\text {an }}\right)$ on $\mathcal{X} \frac{\text { an }}{s^{\prime}}$ are isomorphic.

We set $\mathcal{S}^{\prime}=\operatorname{Spec}\left(\mathcal{O}_{S^{\prime}, s^{\prime}}\right)$ and denote by $\mathcal{S}^{\prime \prime}$ the spectrum of the Henselization of the local ring $\mathcal{O}_{A_{\mathcal{S}}^{1}, \mathbf{s}^{\prime}}$. Since the ring $\mathcal{O}_{S^{\prime}, s^{\prime}}$ is Henselian, there is a canonical 
morphism of schemes

$$
\mathcal{S}^{\prime}=\left\{s^{\prime}, \eta^{\prime}\right\} \rightarrow \mathcal{S}^{\prime \prime}=\left\{s^{\prime \prime}, \eta^{\prime \prime}\right\}
$$

Consider the following commutative diagram with Cartesian squares:

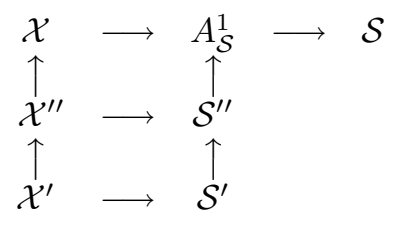

Let $\mathcal{F}^{\prime}$ (resp. $\mathcal{F}^{\prime \prime}$ ) be the inverse image of $\mathcal{F}$ on $\mathcal{X}_{\eta^{\prime}}^{\prime}$ (resp. $\mathcal{X}_{\eta^{\prime \prime}}^{\prime \prime}$ ), and let $\mathcal{K}^{\prime}$ (resp. $\left.\mathcal{K}^{\prime \prime}\right)$ be the fraction field of $\mathcal{O}_{\mathcal{S}^{\prime}, s^{\prime}}\left(\operatorname{resp} . \mathcal{O}_{\mathcal{S}^{\prime \prime}, s^{\prime \prime}}\right)$. (We note that the field $\mathcal{K}^{\prime \prime}$ is quasicomplete because its ring of integers is Henselian.) Let us fix embeddings of fields $\mathcal{K}^{\mathrm{s}} \hookrightarrow \mathcal{K}^{\prime \prime}{ }^{\mathrm{s}} \hookrightarrow \mathcal{K}^{\prime \mathrm{s}}$ over the canonical embeddings $\mathcal{K} \hookrightarrow \mathcal{K}^{\prime \prime} \hookrightarrow \mathcal{K}^{\prime}$. We get a homomorphism of Galois groups $G_{\eta^{\prime}} \rightarrow G_{\eta^{\prime \prime}} \rightarrow G_{\eta}$ and morphisms

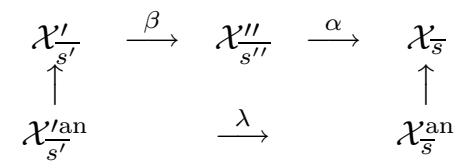

By Lemma 3.4 from $\left[\mathrm{SGA} 4 \frac{1}{2}\right]$, Th. Finitude, there is an isomorphism of sheaves on $\mathcal{X}_{\bar{s}^{\prime \prime}}^{\prime \prime}, \alpha^{*}\left(R^{q} \Psi_{\eta}(\mathcal{F})\right) \stackrel{\sim}{\longrightarrow} R^{q} \Psi_{\eta^{\prime \prime}}\left(\mathcal{F}^{\prime \prime}\right)^{P}$, where $P=G\left(\mathcal{K}^{\prime \prime s} / \mathcal{K}^{\prime \prime n r} \mathcal{K}^{\mathrm{s}}\right)$ (it is a pro$p$-group for $p=\operatorname{char}(k))$. Furthermore, since the formation of vanishing cycles is compatible with any base change (loc. cit., Proposition 3.7), there is an isomorphism of sheaves on $\mathcal{X}_{\bar{s}^{\prime}}^{\prime}, \beta^{*}\left(R^{q} \Psi_{\eta^{\prime \prime}}\left(\mathcal{F}^{\prime \prime}\right)\right) \stackrel{\sim}{\longrightarrow} R^{q} \Psi_{\eta^{\prime}}\left(\mathcal{F}^{\prime}\right)$, compatible with the action of Galois groups. Therefore, there is an isomorphism of sheaves on $\mathcal{X}_{\bar{s}^{\prime}}^{\prime}$, $(\alpha \beta)^{*}\left(R^{q} \Psi_{\eta}(\mathcal{F})\right) \stackrel{\sim}{\longrightarrow} R^{q} \Psi_{\eta^{\prime}}\left(\mathcal{F}^{\prime}\right)^{P}$. Applying the induction hypothesis to the morphism $\mathcal{X}^{\prime} \rightarrow \mathcal{S}^{\prime}$, we get an isomorphism of sheaves on $\mathcal{X}_{\bar{s}^{\prime}}^{\text {an }}$

$$
\lambda^{*}\left(R^{q} \Psi_{\eta}(\mathcal{F})^{\text {an }}\right) \stackrel{\sim}{\longrightarrow} R^{q} \Psi_{\eta^{\prime}}\left(\mathcal{F}^{\prime \text { an }}\right)^{P} .
$$

On the other hand, Corollary 4.4 gives an isomorphism

$$
\lambda^{*}\left(R^{q} \Psi_{\eta}\left(\mathcal{F}^{\mathrm{an}}\right)\right) \stackrel{\sim}{\longrightarrow} R^{q} \Psi_{\eta^{\prime}}\left(\mathcal{F}^{\text {an }}\right)^{Q},
$$

where $Q=G\left(\mathcal{K}^{\prime \mathrm{s}} / \mathcal{K}^{\prime \mathrm{nr}} \mathcal{K}^{\mathrm{s}}\right)$. It is clear that the image of $Q$ under the homomorphism $G_{\eta^{\prime}} \rightarrow G_{\eta^{\prime \prime}}$ is contained in $P$. Thus, Step 2 follows from the following fact.

6.2. Lemma. The homomorphism $Q \rightarrow P$ is surjective.

Proof. Consider the diagram of embeddings of fields

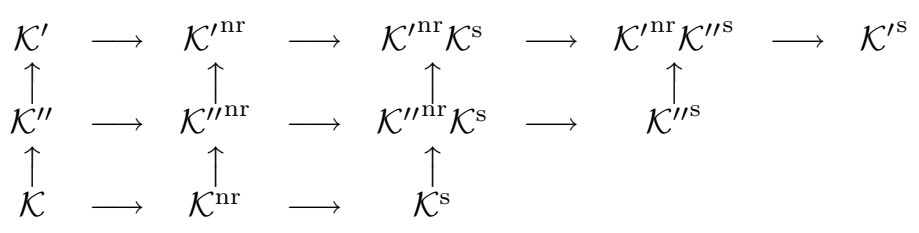


It induces a commutative diagram of homomorphisms of Galois groups

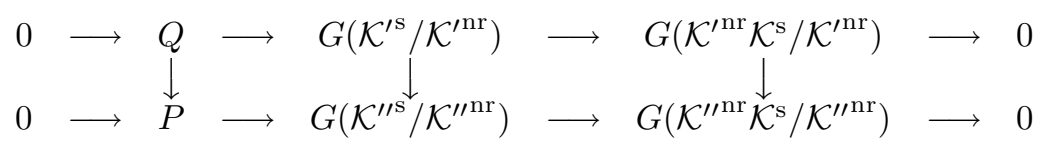

Since the third vertical homomorphism is injective (both groups are subgroups of $G_{\eta}$ ), it suffices to show that the second one is surjective.

Let $\mathcal{L}$ be a finite extension of $\mathcal{K}^{\prime \prime n r}$ in $\mathcal{K}^{\prime \prime \text { s }}$, and let $\mathcal{L}^{\prime}=\mathcal{K}^{\prime n r} \mathcal{L}$. It suffices to verify that

$$
\left[\mathcal{L}: \mathcal{K}^{\prime \prime \mathrm{nr}}\right]=\left[\mathcal{L}^{\prime}: \mathcal{K}^{\prime \mathrm{nr}}\right]
$$

First of all, we remark that since $\mathcal{K}^{\text {nr }}$ and $\mathcal{K}^{\prime \prime n r}$ are quasicomplete discrete valuation fields, then the number on the left hand side is equal to $\left[\left|\mathcal{L}^{*}\right|:\left|\mathcal{K}^{\prime \prime n r *}\right|\right]\left[\widetilde{\mathcal{L}}: \widetilde{\mathcal{K}^{\prime \prime n r}}\right]$, and that on the right hand side is equal to $\left[\left|\mathcal{L}^{* *}\right|:\left|\mathcal{K}^{\prime \text { nr* }}\right|\right]\left[\widetilde{\mathcal{L}^{\prime}}: \widetilde{\mathcal{K}^{\prime n r}}\right]$. One has $\left|\mathcal{K}^{\prime \text { nr* }}\right|=\left|\mathcal{K}^{\prime \prime \text { nr* }}\right|=\left|\mathcal{K}^{*}\right|$. Since $\left|\mathcal{L}^{*}\right| \subset\left|\mathcal{L}^{\prime *}\right|$, it suffices to verify that

$$
\left[\widetilde{\mathcal{L}}: \widetilde{\mathcal{K}^{\prime \prime n r}}\right] \leq\left[\widetilde{\mathcal{L}^{\prime}}: \widetilde{\mathcal{K}^{\prime n r}}\right] .
$$

One has $\widetilde{\mathcal{K}^{\prime n r}}=\kappa\left(s^{\prime}\right)^{\mathrm{s}}$ and $\widetilde{\mathcal{K}^{\prime \prime n r}}=k\left(\mathbf{s}^{\prime}\right)^{\mathrm{s}}$. By Theorem 3.4, the field $\kappa\left(s^{\prime}\right)$ is separable over $k\left(\mathbf{s}^{\prime}\right)$. It follows that $\left[\kappa\left(s^{\prime}\right)^{\mathrm{s}} \widetilde{\mathcal{L}}: \kappa\left(s^{\prime}\right)^{\mathrm{s}}\right]=\left[\widetilde{\mathcal{L}}: k\left(\mathbf{s}^{\prime}\right)^{\mathrm{s}}\right]$. Since $\kappa\left(s^{\prime}\right)^{\mathrm{s}} \widetilde{\mathcal{L}} \subset$ $\widetilde{\mathcal{L}^{\prime}}$, the inequality follows.

Step 3. The homomorphism considered is an isomorphism over all points of $\mathcal{X}_{\bar{s}}^{\mathrm{an}}$.

Since our statement is local, we may assume that $\mathcal{X}$ is affine. After that we may assume that $\mathcal{X}$ is projective over $\mathcal{S}$. Let $\varphi$ denote the canonical morphism $\mathcal{X} \rightarrow \mathcal{S}$. Define a complex $\Delta^{\cdot}$ by the exact triangle in $D_{G_{\eta}}^{+}\left(\mathcal{X}_{\bar{s}}^{\text {an }}\right)$,

$$
\longrightarrow R \Psi_{\eta}(\mathcal{F})^{\text {an }} \longrightarrow R \Psi_{\eta}\left(\mathcal{F}^{\text {an }}\right) \longrightarrow \Delta^{\cdot} \longrightarrow .
$$

We have to show that the cohomology sheaves $H^{q}\left(\Delta^{*}\right)$ of the complex $\Delta^{\cdot}$ are trivial. By Step 2, we know, at least, that they are concentrated at the points of $\mathcal{X}_{\bar{s}}^{\text {an }}$ whose images in $\mathcal{X}_{s}$ are closed points. In particular, these are $\widehat{\mathcal{H}(s)^{a}}$-points of the $\widehat{\mathcal{H}(s)^{a}}$ analytic space $\mathcal{X}_{\bar{s}}^{\text {an }}$. To show that $H^{q}\left(\Delta^{*}\right)=0$, we need the following fact which is a purely non-Archimedean phenomenon.

6.3. Proposition. Suppose that $k$ is algebraically closed. Let $X$ be a Hausdorff $k$-analytic space, and let $F$ be an abelian sheaf on $X$ such that either (1) for any point $x \in X$ with $F_{x} \neq 0$, one has $x \in X(k)$ (as in our situation), or (2) $F$ is torsion with torsion orders prime to $\operatorname{char}(\widetilde{k})$ and, for any point $x \in X$ with $F_{x} \neq 0$, one has $\widetilde{\mathcal{H}(x)}=\widetilde{k}$ and $\left|\mathcal{H}(x)^{*}\right|=\left|k^{*}\right|$ (i.e., $d(x)=0$ in the notation of [Ber1], §9). Then $H_{c}^{q}(X, F)=0$ for all $q \geq 1$. Furthermore, if $H_{c}^{0}(X, F)=0$, then $F=0$.

Proof. Consider the morphism of sites $\pi: X_{\text {ét }} \rightarrow|X|$. By [Ber2], 4.2.4, one has $\left(R^{q} \pi_{*} F\right)_{x}=H^{q}\left(G_{\mathcal{H}(x)}, F_{x}\right), q \geq 0$. If $x \in X(k)$, then the group $G_{\mathcal{H}(x)}$ is trivial. If $\widetilde{\mathcal{H}(x)}=\widetilde{k}$ and $\left|\mathcal{H}(x)^{*}\right|=\left|k^{*}\right|$, then $G_{\mathcal{H}(x)}$ is a $p$-group, where $p=\operatorname{char}(\widetilde{k})$, by [Ber2], 2.4.4. In both cases one has $R^{q} \pi_{*} F=0$ for all $q \geq 1$. Therefore the Leray spectral sequence of the morphism $\pi$ gives an isomorphism $H_{c}^{q}\left(|X|, \pi_{*} F\right) \stackrel{\sim}{\longrightarrow} H_{c}^{q}(X, F)$, $q \geq 0$. Thus, to prove the statement, it suffices to show that the restriction of $F$ to the usual topology of $X$ satisfies the following condition: for any compact subset $\Sigma \subset X$, the canonical homomorphism $F(X) \rightarrow F(\Sigma)$ is surjective (see [God], Ch. II, §3.5). 
Let $f \in F(\Sigma)$. Since the set $\Sigma$ has a basis of paracompact neighborhoods, then, by loc. cit., 3.3.1, $f$ extends to an open neighborhood $\mathcal{U}$ of $\Sigma$. Furthermore, since $\Sigma$ is compact, we can find a finite family of affinoid domains $V_{1}, \ldots, V_{n}$ such that $V=\cup_{i=1}^{n} V_{i} \subset \mathcal{U}$ and $\Sigma \subset \mathcal{V}=\cup_{i=1}^{n} \operatorname{Int}\left(V_{i} / X\right)$. We remark that it suffices to show that

$$
\operatorname{Supp}\left(\left.f\right|_{\mathcal{V}}\right)=\operatorname{Supp}\left(\left.f\right|_{\mathcal{U}}\right) \cap V \text {. }
$$

Indeed, from the equality it follows that the set $\operatorname{Supp}\left(\left.f\right|_{\mathcal{V}}\right)$ is compact, and therefore there exists an element $g \in F(X)$ which is zero outside $\operatorname{Supp}\left(\left.f\right|_{\mathcal{V}}\right)$ and coincides with $f$ on $\mathcal{V}$. The equality follows from the following lemma (the field $k$ in it is not assumed to be algebraically closed).

6.4. Lemma. Any point $x$ of a $k$-analytic space $X$, for which the extension $\widetilde{\mathcal{H}(x)} / \widetilde{k}$ is algebraic and the group $\left|\mathcal{H}(x)^{*}\right| /\left|k^{*}\right|$ is torsion, is contained in the interior of $X$.

Proof. We may assume that $X=\mathcal{M}(\mathcal{A})$ is $k$-affinoid. By [Ber1], 2.5.2, we have to verify that, for any bounded homomorphism $k\left\{r^{-1} T\right\} \rightarrow \mathcal{A}: T \mapsto f$, there exists a polynomial $P=T^{n}+a_{1} T^{n-1}+\cdots+a_{n} \in k[T]$ such that $\left|a_{i}\right| \leq r^{i}$, $1 \leq i \leq n$, and $|P(f)(x)|<r^{n}$. This is evident if $|f(x)|<r$, and therefore we assume that $|f(x)|=r$. One has $r^{m}=|a|$ for some integer $m \geq 1$ and an element $a \in k^{*}$. In particular, $\left|\frac{f^{m}}{a}(x)\right|=1$. Furthermore, we can find a polynomial $Q=T^{l}+a_{1} T^{l-1}+\cdots+a_{l} \in k^{\circ}[T]$ with $\left|Q\left(\frac{f^{m}}{a}\right)(x)\right|<1$. It follows that the polynomial $P(T)=a^{l} Q\left(\frac{T^{m}}{a}\right)$ is the required one.

By Proposition 6.3, to show that $H^{q}\left(\Delta^{\cdot}\right)=0$, it suffices to show that the cohomology of the complex $R \varphi_{\bar{s}_{*}}^{\text {an }}\left(\Delta^{*}\right)$ is trivial or, equivalently, that the canonical morphism $R \varphi_{\bar{s}}^{\text {an }}\left(R \Psi_{\eta}(\mathcal{F})^{\text {an }}\right) \longrightarrow R \varphi_{\bar{s}}^{\text {an }}\left(R \Psi_{\eta}\left(\mathcal{F}^{\text {an }}\right)\right)$ is an isomorphism. By Proposition 4.1(i), the complex on the left hand side is $R \Psi_{\eta}\left(R \varphi_{\eta}^{\text {an }}\left(\mathcal{F}^{\text {an }}\right)\right)$ and, by the similar fact from algebraic geometry and the comparison theorem for cohomology with compact support, the complex on the right hand side is $\left(R \Psi_{\eta}\left(R \varphi_{\eta_{*}} \mathcal{F}\right)\right)^{\text {an }}$. Since $\left(R \varphi_{\eta_{*}} \mathcal{F}\right)^{\text {an }} \stackrel{\sim}{\longrightarrow} R \varphi_{\eta}^{\text {an }}\left(\mathcal{F}^{\text {an }}\right)$ (Proposition 3.3), the required statement follows from the fact that the theorem is true for $\mathcal{S}$ (Step 1). The theorem is proved.

The following statement is deduced from the comparison theorem [Ber2], 7.5.1, and theorem 6.1 in the same way as the theorem [SGA4 $\frac{1}{2}$ ], 1.1, is deduced from the corresponding Theorems [SGA $4 \frac{1}{2}$ ], 1.9 and 3.2 .

6.5. Corollary. Let $\varphi: \mathcal{Y} \rightarrow \mathcal{X}$ be a morphism of finite type between schemes of locally finite type over $\mathcal{S}$ or over $\operatorname{Spec}(\mathcal{A})$, where $\mathcal{M}(\mathcal{A})$ is a one-dimensional regular $k$-affinoid space. Then, for any constructible sheaf $\mathcal{F}$ on $\mathcal{Y}$ with torsion orders prime to char $(\widetilde{k})$ and any $q \geq 0$, there is a canonical isomorphism

$$
\left(R^{q} \varphi_{*} \mathcal{F}\right)^{\text {an }} \stackrel{\sim}{\longrightarrow} R^{q} \varphi_{*}^{\text {an }}\left(\mathcal{F}^{\text {an }}\right) .
$$

\section{$\S 7$. An applichtion}

Let $A$ be a Henselian discrete valuation ring with fraction field $\mathcal{K}$ and algebraically closed residue field $k$, and let $\mathcal{S}=\operatorname{Spec}(A)=\{s, \eta\}$. A finite discrete $G_{\eta}=G_{\mathcal{K}}$-module $\Lambda$ defines on every scheme $\mathcal{Z}$ over $\mathcal{K}$ a finite locally constant sheaf $\Lambda_{\mathcal{Z}}$. We note that any morphism $\varphi: \mathcal{Y} \rightarrow \mathcal{X}$ between schemes of finite type over $\mathcal{S}$ and any closed point $\mathbf{y} \in \mathcal{Y}_{s}$ give rise to homomorphisms of finite abelian 
groups $\theta^{q}(\varphi, \Lambda):\left(R^{q} j_{*} \Lambda_{\mathcal{X}_{\eta}}\right)_{\mathbf{x}} \rightarrow\left(R^{q} j_{*} \Lambda_{\mathcal{Y}_{\eta}}\right)_{\mathbf{y}}$ and $R^{q} \Psi_{\eta}\left(\Lambda_{\mathcal{X}_{\eta}}\right)_{\mathbf{x}} \rightarrow R^{q} \Psi_{\eta}\left(\Lambda_{\mathcal{Y}_{\eta}}\right)_{\mathbf{y}}$, where $\mathbf{x}=\varphi(\mathbf{y})$ and $q \geq 0$. Furthermore, for a scheme $\mathcal{X}$ of finite type over $\mathcal{S}$ and a closed point $\mathbf{x} \in \mathcal{X}_{s}$, let $\widehat{\mathcal{X}}_{/ \mathbf{x}}$ denote the formal completion of $\mathcal{X}$ at $\mathbf{x}$, i.e., $\widehat{\mathcal{X}}_{/ \mathbf{x}}=\operatorname{Spf}\left(\widehat{\mathcal{O}}_{\mathcal{X}, \mathbf{x}}\right)$. It is a formal scheme over $\widehat{\mathcal{S}}=\operatorname{Spf}(\widehat{A})$. Formal schemes considered here are only of this type. The purpose of this section is to prove the following two theorems.

Suppose that $A$ is equicharacteristic, i.e., $\operatorname{char}(\mathcal{K})=\operatorname{char}(k)$.

7.1. Theorem. One can associate homomorphisms

$$
\theta^{q}(\alpha, \Lambda):\left(R^{q} j_{*} \Lambda_{\mathcal{X}_{\eta}}\right)_{\mathbf{x}} \rightarrow\left(R^{q} j_{*} \Lambda_{\mathcal{Y}_{\eta}}\right)_{\mathbf{y}} \text { and } R^{q} \Psi_{\eta}\left(\Lambda_{\mathcal{X}_{\eta}}\right)_{\mathbf{x}} \rightarrow R^{q} \Psi_{\eta}\left(\Lambda_{\mathcal{Y}_{\eta}}\right)_{\mathbf{y}}
$$

with any morphism of formal schemes over $\widehat{\mathcal{S}}, \alpha: \widehat{\mathcal{Y}}_{/ \mathbf{y}} \rightarrow \widehat{\mathcal{X}}_{/ \mathbf{x}}$, and any finite discrete $G_{\eta}$-module $\Lambda$ of order prime to char $(k)$, so that they possess the following properties:

(a) if $\alpha$ is induced by a morphism $\varphi: \mathcal{Y} \rightarrow \mathcal{X}$ over $\mathcal{S}$ with $\varphi(\mathbf{y})=\mathbf{x}$, then $\theta^{q}(\alpha, \Lambda)=\theta^{q}(\varphi, \Lambda)$

(b) if $\beta: \widehat{\mathcal{Z}}_{/ \mathbf{z}} \rightarrow \widehat{\mathcal{Y}}_{/ \mathbf{y}}$ is a similar morphism, then $\theta^{q}(\alpha \beta, \Lambda)=\theta^{q}(\beta, \Lambda) \circ \theta^{q}(\alpha, \Lambda)$;

(c) $\theta^{q}(\alpha, \Lambda)$ is functorial on $\Lambda$.

The second theorem is proved for the schemes $\mathcal{X}$ of finite type over $\mathcal{S}$ satisfying one of the following assumptions:

(1) the generic fiber $\mathcal{X}_{\eta}$ of $\mathcal{X}$ is smooth;

(2) $\mathcal{X}=\mathcal{Z} \times_{\mathcal{R}} \mathcal{S}$, where $\mathcal{R}$ is an algebraic curve over $k, \mathcal{Z} \rightarrow \mathcal{R}$ is a morphism of finite type, and $\mathcal{S} \rightarrow \mathcal{R}$ is a morphism induced by a homomorphism $\mathcal{O}_{\mathcal{R}, s} \rightarrow A$ for which $\widehat{\mathcal{O}}_{\mathcal{R}, s} \stackrel{\sim}{\longrightarrow} \widehat{A}$.

The assumptions (1) and (2) are necessary to apply a result from [Ber3] on the finiteness of the cohomology groups of certain compact $k$-analytic spaces. (Of course, the latter fact should be true for arbitrary compact $k$-analytic spaces, and therefore the assumptions are superfluous.)

7.2. Theorem. Given $\widehat{\mathcal{X}}_{/ \mathbf{x}}$ and $\widehat{\mathcal{Y}}_{/ \mathbf{y}}$, where each of $\mathcal{X}$ and $\mathcal{Y}$ satisfies (1) or (2), and a finite discrete $G_{\eta}$-module $\Lambda$ of order prime to char $(k)$, there exists $n \geq 1$ such that, for any pair of morphisms $\alpha, \beta: \widehat{\mathcal{Y}}_{/ \mathbf{y}} \rightarrow \widehat{\mathcal{X}}_{/ \mathbf{x}}$ over $\widehat{\mathcal{S}}$ that coincide modulo the $n$-th power of the maximal ideal of $\widehat{\mathcal{O}}_{\mathcal{Y}, \mathbf{y}}$, one has $\theta^{q}(\alpha, \Lambda)=\theta^{q}(\beta, \Lambda)$ for all $q \geq 0$.

Proof. By a result of Deligne ([SGA $\left.4 \frac{1}{2}\right]$, Th. Finitude, 3.7), the formation of vanishing cycles is compatible with any base change. Furthermore, since $A$ is Henselian, then the Galois groups of $\mathcal{K}$ and $\widehat{\mathcal{K}}$ coincide (see [Ber2], §2.4), and therefore the canonical morphism from the spectral sequence $\mathcal{H}^{p}\left(I, R^{q} \Psi_{\eta}\left(\Lambda_{\mathcal{X}_{\eta}}\right)\right) \Longrightarrow$ $\bar{i}^{*}\left(R^{p+q} j_{*} \Lambda_{\mathcal{X}_{\eta}}\right)$ over $A$ to the similar spectral sequence over $\widehat{A}$ shows that the sheaves $R^{q} j_{*}\left(\Lambda_{\mathcal{X}_{\eta}}\right)$ do not change if we replace $A$ by its completion. Thus, we may assume that $A$ is complete.

Since $A$ is equicharacteristic, it is isomorphic to $k[[T]]$. We endow the field $k$ with the trivial valuation. The ring $k[[T]]$ is topologically isomorphic to the $k$-affinoid algebra $k\left\{r^{-1} T\right\}$ for any $0<r<1$. In particular, $A=\mathcal{O}(S)=\mathcal{O}_{S, s}$, where $S$ is the open unit disc $D(0 ; 1)$ in $\mathbf{A}^{1}$ with center $s$ at zero. The scheme $\mathcal{X}$ over $A$ gives rise, for each $0<r<1$, to a $k$-analytic space closed over $E(0 ; r)=\mathcal{M}\left(k\left\{r^{-1} T\right\}\right)$. These $k$-analytic spaces are glued together to a $k$-analytic space closed over $S=D(0 ; 1)$ 
which will be denoted by $\mathcal{X}^{\text {an }}$. Shrinking $\mathcal{X}$, we can find regular functions $f_{1}, \ldots, f_{d}$ on $\mathcal{X}$ such that $T, f_{1}, \ldots, f_{d}$ generate the maximal ideal $\mathbf{m}_{\mathbf{x}}$ of $\mathcal{O}_{\mathcal{X}, \mathbf{x}}$. We set

$$
\mathcal{X}_{(x)}^{\text {an }}=\left\{y \in \mathcal{X}^{\text {an }}|| f_{i}(y) \mid<1,1 \leq i \leq d\right\} .
$$

It is an open subset of $\mathcal{X}^{\text {an }}$, and it is clear that it does not change if we shrink $\mathcal{X}$ or replace $f_{1}, \ldots, f_{d}$ by a similar system of elements.

7.3. Lemma. (i) $\mathcal{O}\left(\mathcal{X}_{(x)}^{\mathrm{an}}\right)=\mathcal{O}_{\mathcal{X}^{\mathrm{an}}, x}=\widehat{\mathcal{O}}_{\mathcal{X}, \mathbf{x}}$;

(ii) if $\mathcal{Y}$ is a scheme of finite type over $A$ and $\mathbf{y}$ is a closed point of $\mathcal{Y}_{s}$, then there is a canonical bijection

$$
\operatorname{Hom}_{S}\left(\mathcal{Y}_{(y)}^{\text {an }}, \mathcal{X}_{(x)}^{\text {an }}\right) \stackrel{\sim}{\longrightarrow} \operatorname{Hom}_{A}\left(\widehat{\mathcal{O}}_{\mathcal{X}, \mathbf{x}}, \widehat{\mathcal{O}}_{\mathcal{Y}, \mathbf{y}}\right) .
$$

Proof. If $\mathcal{X}$ is the $d$-dimensional affine space over $A$, then $\mathcal{X}_{(x)}^{\text {an }}$ is the $(d+1)$ dimensional open unit disc in $\mathbf{A}^{d+1}$ with center at zero, and one has

$$
\mathcal{O}\left(\mathcal{X}_{(x)}^{\mathrm{an}}\right)=\mathcal{O}_{\mathcal{X}^{\mathrm{an}}, x}=\widehat{\mathcal{O}}_{\mathcal{X}, \mathbf{x}}=k\left[\left[T, T_{1}, \ldots, T_{d}\right]\right] .
$$

In the general case we can shrink $\mathcal{X}$ and assume that there is a closed immersion $i: \mathcal{X} \rightarrow \mathcal{Z}=\mathbf{A}_{\operatorname{Spec}(A)}^{d}$. Then $\mathcal{X}_{(x)}^{\text {an }}=\mathcal{Z}_{(z)}^{\text {an }} \cap \mathcal{X}^{\text {an }}$, where $\mathbf{z}=i(\mathbf{x})$. Since $\mathcal{Z}_{(z)}^{\text {an }}$ is a Stein space, then the canonical homomorphism $\mathcal{O}\left(\mathcal{Z}_{(z)}^{\text {an }}\right) \rightarrow \mathcal{O}\left(\mathcal{X}_{(x)}^{\text {an }}\right)$ is surjective, and (i) follows. To prove (ii), it suffices to verify that it is true when $\mathcal{X}$ is the $d$-dimensional affine space over $A$. In this case, the left hand side is $\mathcal{O}\left(\mathcal{Y}_{(y)}^{\text {an }}\right)^{d}$ and the right hand side is $\left(\widehat{\mathcal{O}}_{\mathcal{Y}, \mathbf{y}}\right)^{d}$, and therefore the required fact follows from (i).

Theorem 7.1 follows directly from Lemma 7.3, Theorem 6.1 and Corollary 6.5.

Furthermore, since the groups $\left(R^{q} j_{*} \Lambda_{\mathcal{X}_{\eta}}\right)_{\mathrm{x}}$ and $R^{q} \Psi_{\eta}\left(\Lambda_{\mathcal{X}_{\eta}}\right)_{\mathrm{x}}$ are finite and the latter is an inductive limit of $\bar{i}_{\mathcal{L}}^{*}\left(R^{q} j_{\mathcal{L}_{*}} \Lambda_{\mathcal{X}_{\eta}}\right)$, where $\mathcal{L}$ runs through finite extensions of $\mathcal{K}$ in $\mathcal{K}^{\mathrm{s}}$, it suffices to prove Theorem 7.2 only for the groups $\left(R^{q} j_{*} \Lambda_{\mathcal{X}_{\eta}}\right)_{\mathbf{x}}$. Since only finite number among these groups are non-zero, it suffices to prove Theorem 7.2 for each $q$ separately. We set

$$
\mathcal{X}_{(x) \eta}^{\mathrm{an}}=\left\{y \in \mathcal{X}_{(x)}^{\mathrm{an}} \mid T(y) \neq 0\right\} .
$$

7.4. Lemma. If $\mathcal{X}$ satisfies (1) or (2), then there is a canonical isomorphism

$$
H^{q}\left(\mathcal{X}_{(x) \eta}^{\mathrm{an}}, \Lambda\right) \stackrel{\sim}{\longrightarrow}\left(R^{q} j_{*} \Lambda_{\mathcal{X}_{\eta}}\right)_{\mathbf{x}} .
$$

Proof. For $0<r<1$, we set

$$
E(x ; r)=\left\{y \in \mathcal{X}_{(x)}^{\mathrm{an}}|| T(y)|\leq r,| f_{i}(y) \mid \leq r, 1 \leq i \leq d\right\} .
$$

It is an affinoid neighborhood of the point $x$ in $\mathcal{X}_{(x)}^{\text {an }}$. We also set $E_{\eta}(x ; r)=\{y \in$ $E(x ; r) \mid T(y) \neq 0\}$. By Theorem 6.1, there is a canonical isomorphism

$$
\lim _{r \rightarrow 0} H^{q}\left(E_{\eta}(x ; r), \Lambda\right) \stackrel{\sim}{\longrightarrow}\left(R^{q} j_{*} \Lambda_{\mathcal{X}_{\eta}}\right)_{\mathbf{x}} .
$$

We will prove that for any $0<r<1$ there exists $0<r^{\prime} \leq r\left(\right.$ resp. $\left.r \leq r^{\prime \prime}<1\right)$ such that the image of $H^{q}\left(E_{\eta}(x ; r), \Lambda\right)$ in $H^{q}\left(E_{\eta}\left(x ; r^{\prime}\right), \Lambda\right)\left(\operatorname{resp} . H^{q}\left(E_{\eta}\left(x ; r^{\prime \prime}\right), \Lambda\right)\right.$ in $\left.H^{q}\left(E_{\eta}(x ; r), \Lambda\right)\right)$ maps isomorphically onto $\left(R^{q} j_{*} \Lambda_{\mathcal{X}_{\eta}}\right)_{\mathbf{x}}$. This will imply the required fact because, by [Ber2], 6.3.12, this will give an isomorphism

$$
H^{q}\left(\mathcal{X}_{(x) \eta}^{\text {an }}, \Lambda\right) \stackrel{\sim}{\longrightarrow} \lim _{r \rightarrow 1} H^{q}\left(E_{\eta}(x ; r), \Lambda\right) \stackrel{\sim}{\longrightarrow}\left(R^{q} j_{*} \Lambda_{\mathcal{X}_{\eta}}\right)_{\mathbf{x}}
$$


If $\mathcal{X}$ satisfies (1), then $\mathcal{X}_{(x)}^{\text {an }} \eta$ is a smooth $k$-analytic space. If $\mathcal{X}$ satisfies $(2)$, then $\mathcal{X}_{(x)}^{\text {an }}$ is an open analytic domain in the analytification of the scheme $\mathcal{Z}$ over $k$. In both cases, Corollary 5.6 from [Ber3] implies that for any compact analytic domain $V \subset \mathcal{X}_{(x)_{\eta}}^{\text {an }}$ the group $H^{q}(V, \Lambda)$ is finite. For $0<r^{\prime} \leq r$, we set $A\left(x ; r^{\prime}, r\right)=\{y \in$ $\left.E(x ; r)|| T(y) \mid \geq r^{\prime}\right\}$ (it is an affinoid domain in $\left.\mathcal{X}_{(x)}^{\text {an }}\right)$. Since $E_{\eta}(x ; r)$ is a union of $A\left(x ; r^{\prime}, r\right)$ over all $0<r^{\prime} \leq r$, then, by [Ber2], 6.3.12, the group $H^{q}\left(E_{\eta}(x ; r), \Lambda\right)$ is a projective limit of the finite groups $H^{q}\left(A\left(x ; r^{\prime}, r\right), \Lambda\right)$, and, in particular, it can be endowed with the structure of a profinite group.

Let $r_{0}$ be such that the homomorphism $H^{q}\left(E_{\eta}\left(x ; r_{0}\right), \Lambda\right) \rightarrow\left(R^{q} j_{*} \Lambda_{\mathcal{X}_{\eta}}\right)_{\mathbf{x}}$ is surjective and denote by $P$ its kernel. For $0<t \leq r_{0}$, let $P_{t}$ denote the kernel of the continuous homomorphism $H^{q}\left(E_{\eta}\left(x ; r_{0}\right), \Lambda\right) \rightarrow H^{q}\left(E_{\eta}(x ; t), \Lambda\right)$. We claim that there exists $0<t \leq r_{0}$ such that the subgroup $P_{t}$ has finite index. Indeed, assume that this is not true. The closure $\bar{P}$ of $P$ is an open subgroup of $H^{q}\left(E_{\eta}\left(x ; r_{0}\right), \Lambda\right)$, and the subgroups $P_{t}$ are nowhere dense in $\bar{P}$. Let $g_{1}, \ldots, g_{m}$ be representatives of the cosets of $P$ in $\bar{P}$, and let $t_{1}, t_{2}, \ldots$ be an arbitrary sequence of positive numbers with $t_{j} \leq r_{0}$ and $t_{j} \rightarrow 0$ as $j \rightarrow \infty$. Then the compact space $\bar{P}$ is a union of the countable family of the nowhere dense subsets $g_{i}+P_{t_{j}}$. By the classical Baire Theorem (see [Kel], Ch. 6, Theorem 34), this is impossible.

Thus, we can find a number $0<r_{0}^{\prime} \leq r_{0}$ such that the image of $H^{q}\left(E_{\eta}\left(x ; r_{0}\right), \Lambda\right)$ in $H^{q}\left(E_{\eta}\left(x ; r_{0}^{\prime}\right), \Lambda\right)$ is finite. We can even decrease $r_{0}^{\prime}$ and assume that this image maps isomorphically onto $\left(R^{q} j_{*} \Lambda_{\mathcal{X}_{\eta}}\right)_{\mathbf{x}}$. Let $t_{0}$ be the number with $r_{0}^{\prime}=r_{0}^{t_{0}}$. We claim that any $0<r<1$ possesses the above property with $r^{\prime}=r^{t_{0}}$. (This will give the required fact for $r^{\prime \prime}=r^{t_{0}^{-1}}$.)

Let $t$ be a positive number. Then for any $k$-affinoid algebra $\mathcal{A}$ the Banach $k$ algebra $\mathcal{A}^{t}$, which coincides with $\mathcal{A}$ and is endowed with the $t$-th power of the norm on $\mathcal{A}$, is also $k$-affinoid. This gives rise to a functor $\Phi_{t}: X=\mathcal{M}(\mathcal{A}) \mapsto X^{t}=\mathcal{M}\left(\mathcal{A}^{t}\right)$ from the category of $k$-affinoid spaces to itself, and one has $\Phi_{t} \circ \Phi_{t^{\prime}}=\Phi_{t t^{\prime}}$. The functors $\Phi_{t}: X \mapsto X^{t}$ extend in the evident way to the whole category of $k$-analytic spaces, and one has $\Phi_{t} \circ \Phi_{t^{\prime}} \stackrel{\sim}{\longrightarrow} \Phi_{t t^{\prime}}$. If $Y \rightarrow X$ is an étale morphism, then the induced morphism $Y^{t} \rightarrow X^{t}$ is also étale. In this way we get an isomorphism of sites $X_{\text {ét }} \stackrel{\sim}{\longrightarrow} X_{\text {ét }}^{t}$ that induces an isomorphism $F \mapsto F^{t}$ between the corresponding topoi. In particular, for any étale abelian sheaf $F$ on $X$ there is a canonical isomorphism $H^{q}(X, F) \stackrel{\sim}{\longrightarrow} H^{q}\left(X^{t}, F^{t}\right)$.

For example, in our situation one has $E_{\eta}(x ; r)^{t} \stackrel{\sim}{\longrightarrow} E_{\eta}\left(x ; r^{t}\right)$, and therefore if the image of $H^{q}\left(E_{\eta}\left(x ; r_{0}\right), \Lambda\right)$ in $H^{q}\left(E_{\eta}\left(x ; r_{0}^{t_{0}}\right), \Lambda\right)$ maps isomorphically onto $\left(R^{q} j_{*} \Lambda\right)_{\mathbf{x}}$, then the same is true for $r_{0}^{t}$ instead of $r_{0}$. Since $\left\{r_{0}^{t} \mid t>0\right\}=\{0<r<1\}$, we get our claim and the lemma.

Fix a number $0<r<1$. Given $\widehat{\mathcal{X}}_{/ \mathbf{x}}$ and $\widehat{\mathcal{Y}}_{/ \mathbf{y}}$ as in Theorem 7.2 , we can find, by Lemma 7.4 and its proof, a number $0<r^{\prime} \leq r$ such that the canonical homomorphisms $H^{q}\left(\mathcal{X}_{(x)}^{\text {an }}, \Lambda\right) \rightarrow H^{q}\left(A\left(x ; r^{\prime}, r\right), \Lambda\right)$ and $H^{q}\left(\mathcal{Y}_{(y)}^{\text {an }}, \Lambda\right) \rightarrow H^{q}\left(A\left(y ; r^{\prime}, r\right), \Lambda\right)$ are injective. We now apply Theorem 7.1 from [Ber3] to the $k$-affinoid space $A\left(x ; r^{\prime}, r\right)$. Since the functions $f_{1}, \ldots, f_{d}, \frac{1}{T}$ form a $k$-affinoid generating system for the $k$-affinoid algebra $\mathcal{O}\left(A\left(x ; r^{\prime}, r\right)\right)$, it follows that there exist $t_{1}, \ldots, t_{d}>0$ such that for any pair of morphisms of Hausdorff $k$-analytic spaces $\varphi, \psi: Y \rightarrow A\left(x ; r^{\prime}, r\right)$ over $S$ with $\rho\left(\varphi^{*} f_{i}-\psi^{*} f_{i}\right) \leq t_{i}, 1 \leq i \leq d$, the homomorphisms from the image of $H^{q}\left(\mathcal{X}_{(x)}^{\text {an }}, \Lambda\right)$ in $H^{q}\left(A\left(x ; r^{\prime}, r\right), \Lambda\right)$ to $H^{q}(Y, \Lambda)$ induced by $\varphi$ and $\psi$ coincide. Let 
$n \geq 1$ be an integer with $r^{n} \leq t_{i}, 1 \leq i \leq d$. Then, for any pair of morphisms $\alpha, \beta: \widehat{\mathcal{Y}}_{/ \mathbf{y}} \rightarrow \widehat{\mathcal{X}}_{/ \mathbf{x}}$ over $\widehat{\mathcal{S}}$ that coincide modulo the $n$-th power of the maximal ideal of $\widehat{\mathcal{O}}_{\mathcal{Y}, \mathbf{y}}$, one has $\rho\left(\alpha^{*} f_{i}-\beta^{*} f_{i}\right) \leq t_{i}$ on $A\left(y ; r^{\prime}, r\right)$. It follows that the homomorphisms from $H^{q}\left(\mathcal{X}_{(x)}^{\text {an }}, \Lambda\right) \rightarrow H^{q}\left(\mathcal{Y}_{(y)_{\eta}}^{\text {an }}, \Lambda\right)$ induced by $\alpha$ and $\beta$ coincide, and therefore $\theta^{q}(\alpha, \Lambda)=\theta^{q}(\beta, \Lambda)$. Theorem 7.2 is proved.

Given $\widehat{\mathcal{X}}_{/ \mathbf{x}}$, let $\mathcal{G}\left(\widehat{\mathcal{X}}_{/ \mathbf{x}}\right)$ denote the group of automorphisms of the formal scheme $\widehat{\mathcal{X}}_{/ \mathbf{x}}$ over $\widehat{\mathcal{S}}$. By Theorem 7.1, the group $\mathcal{G}\left(\widehat{\mathcal{X}}_{/ \mathbf{x}}\right)$ acts on the finite groups $\left(R^{q} j_{*} \Lambda_{\mathcal{X}_{\eta}}\right)_{\mathbf{x}}$ and $R^{q} \Psi_{\eta}\left(\Lambda_{\mathcal{X}_{\eta}}\right)_{\mathbf{x}}$. Furthermore, for $n \leq 1$, let $\mathcal{G}_{n}\left(\widehat{\mathcal{X}}_{/ \mathbf{x}}\right)$ denote the subgroup of $\mathcal{G}\left(\widehat{\mathcal{X}}_{/ \mathbf{x}}\right)$ consisting of the automorphisms that are trivial modulo the $n$-th power of the maximal ideal of $\widehat{\mathcal{O}}_{\widehat{\mathcal{X}}, \mathbf{x}}$. By Lemma 8.7 from [Ber3] the groups $\mathcal{G}_{n}\left(\mathcal{X}_{/ \mathbf{x}}\right), n \geq 2$, are uniquely $l$-divisible for any prime $l \neq \operatorname{char}(k)$. The following statement easily follows from this fact and Theorem 7.1.

7.5. Corollary. Given $\widehat{\mathcal{X}}_{/ \mathbf{x}}$, where $\mathcal{X}$ satisfies (1) or (2), and a finitely generated $\mathbf{Z}_{l}$-module $\Lambda, l \neq \operatorname{char}(k)$, endowed with a continuous action of $G_{\eta}$, there exists $n \geq 1$ such that $\mathcal{G}_{n}\left(\widehat{\mathcal{X}}_{/ \mathbf{x}}\right)$ acts trivially on all of the groups $\left(R^{q} j_{*}\left(\Lambda / l^{m} \Lambda\right)_{\mathcal{X}_{\eta}}\right)_{\mathbf{x}}$ and $\left(R^{q} \Psi_{\eta}\left(\Lambda / l^{m} \Lambda\right)_{\mathcal{X}_{\eta}}\right)_{\mathbf{x}}, q \geq 0, m \geq 0$.

7.6. Remark. Laumon proved a statement similar to that of Corollary 7.5 for the action of the automorphism group of the Henselization of $\mathcal{X}$ at $\mathbf{x}$ on $R^{q} \Psi_{\eta}\left(\Lambda_{\mathcal{X}_{\eta}}\right)_{\mathbf{x}}$ under the assumption that the morphism $\mathcal{X} \rightarrow \mathcal{S}$ is smooth outside $\mathbf{x}$ (see [Lau], p. $34,6.3 .1)$. In the case when $A$ is of mixed characteristic, Brylinski proved a similar statement under the assumptions that the morphism $\mathcal{X} \rightarrow \mathcal{S}$ is of relative dimension one and $\mathcal{X}_{\eta}$ is smooth (see [Bry]).

\section{REFERENCES}

[Ber1] V. G. Berkovich, Spectral theory and analytic geometry over non-Archimedean fields, Mathematical Surveys and Monographs, vol. 33, Amer. Math. Soc., Providence, RI, 1990. MR 91k:32038

[Ber2] _ Étale cohomology for non-Archimedean analytic spaces, Publ. Math. IHES 78 (1993), 5-161. MR 95c:14017

[Ber3] , Vanishing cycles for formal schemes, Invent. Math. 115 (1994), 539-571. MR 95f: 14034

[Ber4] On the comparison theorem for étale cohomology of non-Archimedean analytic spaces, Israel J. Math. 92 (1995), 45-60. CMP 96:03

[Bry] J. L. Brylinski, Un lemme sur les cycles évanescents en dimension relative 1, Ann. Scient. Éc. Norm. Sup. 19 (1986), 460-467.

[God] R. Godement, Topologie Algébrique et Théorie des Faisceaux, Hermann, Paris, 1958. MR 21:1583

[EGA1] A. Grothendieck and J. Dieudonné, Eléments de Géométrie Algébrique. I. Le langage des schémas, Springer, Berlin-Heidelberg-New York, 1971. MR 36:177a; MR 29:1207

[EGA4] Eléments de Géométrie Algébrique. IV. Étude local des schémas et des morphismes de schémas, Publ. Math. IHES, 20 (1964), 24 (1965), 28 (1966), 32 (1967). MR 30:3885; MR 33:7330; MR 36:178; MR 39:220

[Kel] J. L. Kelley, General Topology, D. Van Nostrand Company, Toronto-London-New York, 1955. MR 16:1136c

[Lau] G. Laumon, Charactéristique d'Euler-Poincaré et sommes exponentielles, Thése, Université de Paris-Sud, Orsay, 1983.

[SGA1] A. Grothendieck, Revêtements Étales et Groupe Fondamental, Lecture Notes in Math. 224, Springer, Berlin-Heidelberg-New York, 1971. MR 50:7129 
[SGA4] M. Artin, A. Grothendieck, and J.-L. Verdier, Théorie des Topos et Cohomologie Étale des Schémas, Lecture Notes in Math. 269, 270, 305, Springer, BerlinHeidelberg-New York, 1972-1973. MR 50:7130; MR 50:7131; MR 50:7132

[SGA $\left.4 \frac{1}{2}\right] \quad$ P. Deligne et al., Cohomologie Étale, Lecture Notes in Math. 569, Springer, BerlinHeidelberg-New York, 1977. MR 57:3132

[SGA7] A. Grothendieck, P. Deligne, and N. Katz, Groupes de Monodromie en Géométrie Algéb-rique, Lecture Notes in Math. 288, 340, Springer, Berlin-Heidelberg-New York, 1972-1973. MR 50:7134; MR 50:7135

Department of Theoretical Mathematics, The Weizmann Institute of Science, P.O.B. 26, 76100 ReHovot, ISRAEL

E-mail address: vova@wisdom.weizmann.ac.il 